\title{
Cavity optomechanics and cooling nanomechanical oscillators using microresonator enhanced evanescent near-field coupling
}

\section{Optomécanique en cavité et refroidissement de nanorésonateurs mécaniques par couplage en champ proche à un microtore}

\author{
G. Anetsberger ${ }^{\text {a }}$, E.M. Weig ${ }^{b}$, J.P. Kotthaus ${ }^{b}$, T.J. Kippenberg ${ }^{\text {a,c, } *}$ \\ ${ }^{a}$ Max-Planck-Institut für Quantenoptik, Hans-Kopfermann-Str. 1, 85748 Garching, Germany \\ ${ }^{\mathrm{b}}$ Fakultät für Physik and Center for NanoScience (CeNS), Ludwig-Maximilians-Universität (LMU), Geschwister-Scholl-Platz 1, 80539 München, Germany \\ c École polytechnique fédérale de Lausanne, EPFL, Ch-1015 Lausanne, Switzerland
}

Keywords:

Nanomechanical oscillator

Precision measurement

Cavity optomechanics

Microresonator

Dynamical backaction

Radiation pressure cooling and amplication

Mots-clés:

Nanorésonateur mécanique

Mesure de précision

\begin{abstract}
A B S T R A C T
Nanomechanical oscillators are at the heart of a variety of precision measurements. This article reports on dispersive radiation coupling of nanomechanical oscillators to the evanescent near-field of toroid optical microresonators. The optomechanical coupling coefficient which reaches values $>200 \mathrm{MHz} / \mathrm{nm}$, corresponding to a vacuum optomechanical coupling rate $>4 \mathrm{kHz}$, is characterized in detail and good agreement between experimental, analytical and finite element simulation based values is found. It is shown that both the mode-structure and -patterns of nanomechanical oscillators can be characterized relying solely on Brownian motion. Moreover, it is demonstrated that the radiation pressure interaction can cause self-sustained coherent nanomechanical oscillations at nano-Watt power levels as well as cooling of the nanomechanical oscillator. Finally, the feasibility of coupling nanomechanical motion to two optical modes where the optical mode spacing exactly equals the mechanical resonance frequency is demonstrated for the first time. As shown here, this Raman-type scheme allows both amplification and cooling.
\end{abstract}

\section{R É S U M É}

Les nanorésonateurs mécaniques sont au coeur de nombreuses mesures de précision. Nous avons obtenu un couplage dispersif par pression de radiation entre un nanorésonateur et le champ évanescent au voisinage d'un microrésonateur en forme de torö̈de. Le coefficient de couplage optomécanique atteint dans ce système une valeur supérieure à $200 \mathrm{MHz} / \mathrm{nm}$, correspondant à un décalage supérieur à $4 \mathrm{kHz}$ associé aux fluctuations quantiques de position du nanorésonateur. La caractérisation détaillée de ce couplage montre un bon accord entre l'expérience et les valeurs déterminées analytiquement ou par simulation par éléments finis. Nous montrons que la structure du mode mécanique du nanorésonateur peut être déterminée à partir de la seule observation de son mouvement brownien. De plus, nous avons observé que l'interaction par pression de radiation peut conduire à des oscillations cohérentes et auto-entretenues du nanorésonateur pour des puissances de l'ordre du nanowatt, et aussi à un refroidissement du nanorésonateur. Enfin, la possibilité de coupler le mouvement du nanorésonateur à deux modes optiques dont l'espacement en fréquence correspond exactement à la fréquence de résonance mécanique est démontrée pour la

\footnotetext{
* Corresponding author at: École polytechnique fédérale de Lausanne, EPFL, Ch-1015 Lausanne, Switzerland. E-mail address: tobias.kippenberg@epfl.ch (T.J. Kippenberg).
} 
première fois. Nous montrons que ce mécanisme de type Raman permet à la fois une amplification et un refroidissement du nanorésonateur.

\section{Introduction}

In recent years, the combination of optical microcavities and micromechanical systems has triggered a rapid development of the research field cavity optomechanics [1-3]. Most generally speaking, this field of research investigates the interaction of light with a mechanical oscillator via radiation pressure where the strength of the interaction is enhanced using an optical cavity. A particularly intriguing aspect of this field is the prospect of realizing quantum mechanical experiments which have so far been restricted to cold gases or trapped ions with tangible mechanical oscillators. This would open up a new arena for quantum physics and quantum engineering. In 2005, it was shown that in fact the radiation pressure of light can be used to drive laser-like oscillations $[4,5]$ of a micromechanical oscillator, also referred to as "phonon lasing". The application of such compact, low-power photonic oscillators as frequency references is currently investigated [6-8]. Subsequently, it has been demonstrated that micromechanical oscillators may be efficiently cooled by radiation pressure [9-12]. In particular, using suitable quantum optomechanical treatment, the feasibility of ground state cooling using radiation pressure has been theoretically shown $[13,14]$. Subsequently, a variety of researchers pursued experiments aiming at initializing a solid-state mechanical oscillator in its quantum-ground state using radiation pressure cooling [15-21]. This would provide a novel route to the new regime of quantum physics dealing with tangible mechanical oscillators which has recently been entered by conventional refrigeration [22]. Just as reaching the ultimate quantum limits in displacement detection and thus maximizing the performance of mechanical sensors of mass [23], charge [24] and magnetic field [25], this is an ongoing and very actively pursued paradigm in the field of cavity optomechanics.

Until 2009, the vast majority of cavity optomechanical systems relied on Fabry-Perot type resonator configurations where a light beam is trapped between a fixed and a movable mirror. This experimental setting has its origin in laser gravitational wave interferometers and enabled extremely sensitive measurements of the cavity length variations down to a sensitivity of $10^{-19} \mathrm{~m} / \sqrt{\mathrm{Hz}}$ already in the 1990s [26,27]. In the subsequent decade, advances in microfabrication have, moreover, allowed miniaturization of the employed cavity mirrors and thus a concomitant drastic increase of their susceptibility to external forces. This lead to the observation of dynamic radiation pressure phenomena that were predicted already decades ago, such as radiation pressure cooling of micromechanical oscillators [9-12]. Using the aforementioned experimental configuration, the miniaturization of the resonators is, however, fundamentally bound by the optical diffraction limit. The reflecting element must not be smaller than the diffraction limited optical spot size in order to prevent diffraction from degrading the optical quality factor of the employed cavity. Thus, this approach does not allow straightforward extension of quantumlimited measurements of mechanical motion or radiation pressure actuation to nanomechanical oscillators.

Such an extension, in turn, would be interesting from several points of view. First, nanomechanical oscillators have been successfully used as ultra-sensitive probes of mass and external forces, allowing single atomic-mass resolution [23] or single electron-spin detection [25] despite the fact that the employed detection methods were partially far from being quantumlimited. Improved and possibly quantum-limited transduction of nanomechanical motion could thus lead to further leaps in the performance of nanomechanical oscillators as sensitive probes. Second, the fact that in recent years nanomechanical elements that combine high mechanical quality, i.e. low coupling to the thermal environment, with extremely small effective mass (when compared to micromechanical elements) have been developed, makes them highly interesting for probing quantum limits of mechanical motion.

In this article an approach based on cavity-enhanced optical near-fields (schematically shown in Fig. 1) will be reported that enabled extending quantum-limited cavity optomechanical coupling to nanomechanical oscillators [28]. This paper moreover reports for the first time the measurement of nanomechanical motion with an imprecision at the level of the standard quantum limit [28], a long sought-after goal for nanomechanical oscillators [30-36], as shown in Fig. 2 (we note that such imprecision has been obtained prior only for large scale mechanical oscillators). The employed approach circumvents the optical diffraction limit by resorting to evanescent optical near-fields which have also been very successfully used in the domain of near-field microscopy. Already in 1983, Braginsky and Vyatchanin envisioned to use the evanescent near-field of an optical whispering-gallery resonator to couple to a dielectric "stick" parallel to the propagation direction of the optical field [37]. Several years later, around 1990, the optical forces between microspheres evanescently coupled to each other were studied both experimentally and theoretically [38]. In the light of the advances in microfabrication, the idea of near-field coupling was then, during the last five years, extended to optical waveguides [39-41]. The mechanical element and the photons in such a near-field coupling configuration exchange momentum via the optical gradient force, as opposed to the scattering force used in the traditional Fabry-Perot setups. The pioneering work of Ashkin [42] experimentally demonstrated that optical gradient fields can be used to exert considerable forces on particles and indeed provide a powerful tool for a variety of applications, complementing the capabilities of the scattering force for cooling or amplifying a solid state mechanical oscillator's motion [9] or for cooling atoms and ions [43]. In Ref. [44], a recent overview of the optical gradient force and its applications in the context of optomechanical systems is given.

The first experimental implementation of a nanomechanical element whose motion is actuated and transduced by optical gradient fields was reported in 2008 [47]. In 2009, the first cavity-enhanced systems incorporating a nanomechanical 

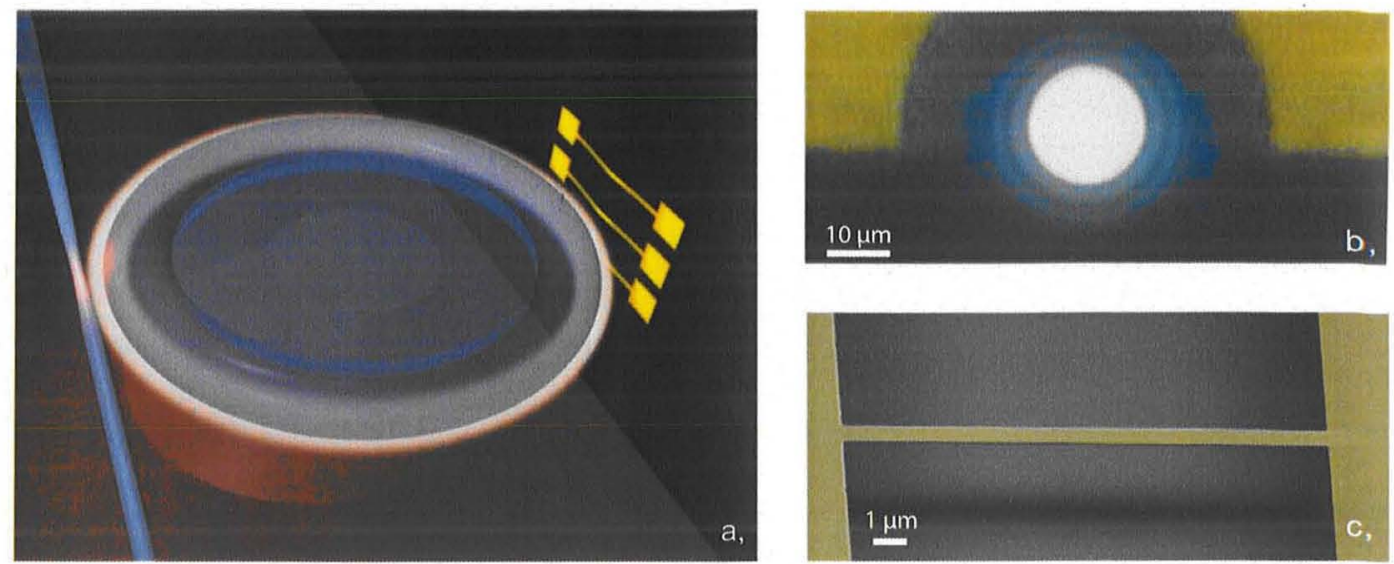

Fig. 1. Schematic of the experimental principle, a, A toroid microresonator is pumped using a tapered optical fibre. The near-field of the optical cavity mode allows dispersive coupling to nanomechanical oscillators, here in the form of nanomechanical strings. $b$, Microscope top view of a toroid microresonator $(R=16 \mu \mathrm{m})$ suitable for near-field probing, as explained in the text. c, (False colour) Scanning electron micrograph of a typical silicon nitride string used for the experiments shown in this chapter $\left(15 \times 0.5 \times 0.1 \mu^{3}\right)$.
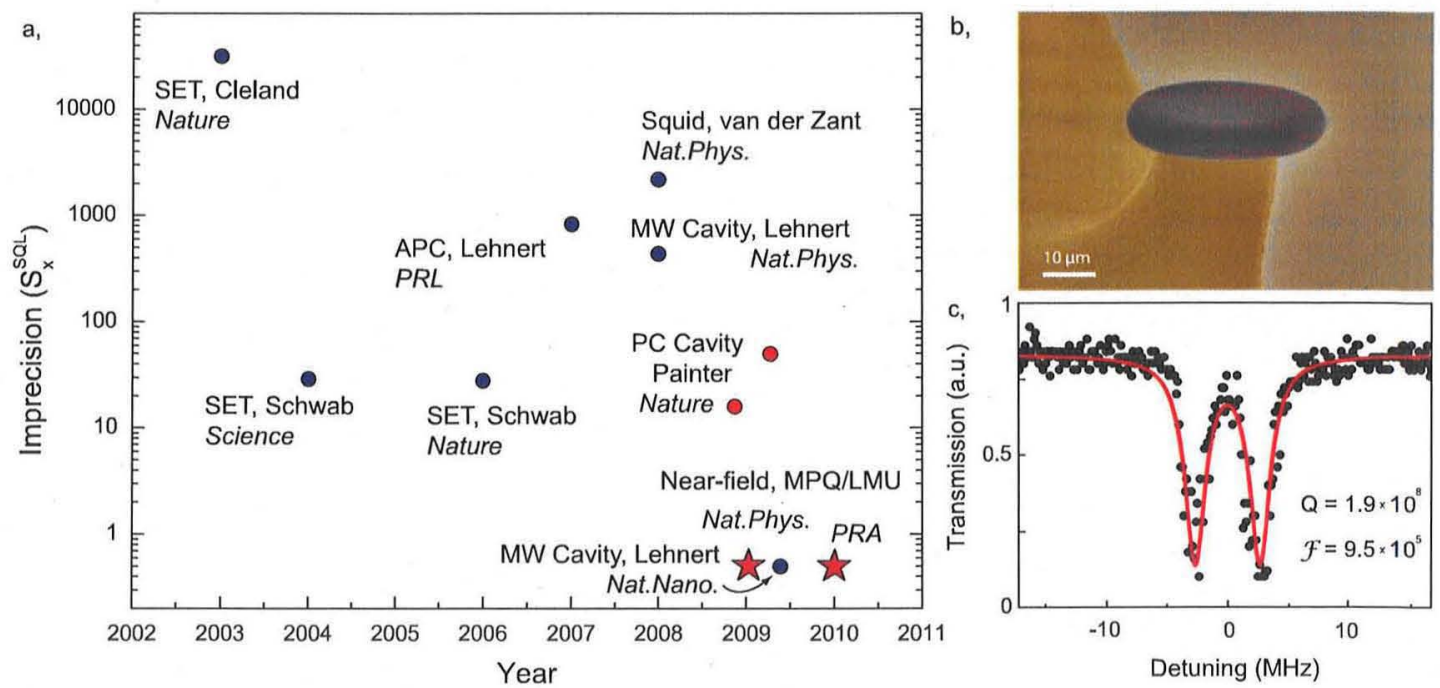

Fig. 2. a, Measurement imprecision of nanomechanical motion approaching and bypassing the level of the SQL in the past years, using various techniques. The blue data points represent experiments requiring cryogenic operation, performed in the groups of Cleland [24], Schwab [31,32], Lehnert [33,34,45] and van der Zant [35] (SET: single-electron transistor, APC: atomic point contact, Squid: superconducting quantum interference device, MW: microwave). The red data points correspond to systems operating at room temperature realized in the group of Painter [36,46] (PC: photonic crystal) and using the near-field approach reported here $[28,29]$. The near-field approach (stars) allowed for the first time a measurement imprecision below the level of the SQL in 2009 [28] and a 100 times reduction of necessary input power in 2010 [29]. It is based on toroid near-field sensors (b, false colour scanning electron micrograph) which allow ultra-high optical $Q\left(Q=1.9 \times 10^{8}\right.$ in the case of c, corresponding to a finesse of $\left.\mathcal{F}=0.95 \times 10^{6}\right)$.

element were studied using a photonic crystal structure [36] and the system reported here: a toroid microresonator evanescently coupled to a nanomechanical string [28].

A schematic of the experimental system is shown in Fig. 1. Bringing nanomechanical oscillators which are fabricated on a silicon chip into the vicinity of the optical modes of a toroid - which rests on a separate chip - requires the toroid to be fabricated at the edge of its chip support such that the silica torus extends across the silicon chip. Such a toroid is shown in Fig. 2b (see also Fig. 1b). These devices can be fabricated with similarly high finesse $\left(\sim 10^{6}\right)$ as regular toroids, as shown in Fig. 2c, and probed by a tapered fibre. A three axis piezo cube allows both translational fine- and coarse-positioning of the nanomechanical oscillators. The respective rotational degrees of freedom are fine-tuned manually. All experiments are carried out in vacuum to rule out gas damping of the nanomechanical oscillators. The optomechanical coupling region is however designed sufficiently close to a vacuum window to allow optical access by a long working distance microscope. 
Table 1

Overview of recent cavity optomechanical systems (*requiring operation in a dilution refrigerator). The near-field platform (italics) displays a vacuum optomechanical coupling rate $g_{0}$ that exceeds the mechanical damping rate $\Gamma_{\mathrm{m}}$ as well as large zero-point motion $x_{\mathrm{zpf}}$.

\begin{tabular}{|c|c|c|c|c|c|c|}
\hline System & $g_{0} / 2 \pi[\mathrm{Hz}]$ & $\kappa / 2 \pi[\mathrm{Hz}]$ & $\Gamma_{\mathrm{m}} / 2 \pi[\mathrm{Hz}]$ & $\Omega_{\mathrm{m}} / \kappa$ & $x_{\mathrm{zpf}}[\mathrm{fm}]$ & Ref. \\
\hline Crystalline microresonator & 1 & $2 \times 10^{5}$ & $1 \times 10^{3}$ & 12 & 0.003 & [50] \\
\hline Movable mirror & $2 \times 10^{-3}$ & $2 \times 10^{4}$ & 0.06 & 0.01 & 0.01 & [51] \\
\hline Microsphere & $1 \times 10^{3}$ & $3 \times 10^{7}$ & $8 \times 10^{4}$ & 5 & 0.04 & [36] \\
\hline Micromirror & 1 & $2 \times 10^{6}$ & 80 & 0.4 & 0.01 & {$[10]$} \\
\hline Micromirror & 5 & $8 \times 10^{5}$ & 30 & 1 & 0.5 & [52] \\
\hline Micromirror & 300 & $8 \times 10^{8}$ & 600 & 0.001 & 40 & [53] \\
\hline Spoke-microresonator & 500 & $5 \times 10^{6}$ & 500 & 5 & 0.2 & [54] \\
\hline Membrane-in-the-middle & 5 & $2 \times 10^{5}$ & 0.1 & 0.6 & 1 & [16] \\
\hline Double-microdisk & $8 \times 10^{4}$ & $1 \times 10^{8}$ & $2 \times 10^{3}$ & 0.06 & 3 & [55] \\
\hline Optomechanical crystal & $2 \times 10^{5}$ & $5 \times 10^{9}$ & $2 \times 10^{6}$ & 0.5 & 3 & [46] \\
\hline Photonic crystal cavity & $6 \times 10^{5}$ & $2 \times 10^{9}$ & $8 \times 10^{4}$ & 0.004 & 5 & [36] \\
\hline Nanomechanical rod & - & $8 \times 10^{8}$ & 300 & 0.002 & - & [56] \\
\hline Near-field nanomechanics & 500 & $5 \times 10^{6}$ & 100 & 2 & 20 & [29] \\
\hline Near-field nanomechanics & 50 & $2 \times 10^{9}$ & $5 \times 10^{4}$ & 0.02 & 20 & {$[57]$} \\
\hline Microwave nanomechanics* & 1 & $3 \times 10^{6}$ & 10 & 0.4 & 30 & [45] \\
\hline Microwave nanomechanics* & 2 & $6 \times 10^{5}$ & 6 & 2 & 30 & [21] \\
\hline
\end{tabular}

\section{Optomechanical coupling - Studied systems and their parameters}

Typically, the optomechanical coupling between an electromagnetic resonator and a mechanical oscillator is linearized for small fluctuations $x$ of the mechanical oscillator around its equilibrium position $x_{0}$. The optical resonance frequency $\omega\left(x_{0}\right)$ which may depend on the position $x_{0}$ of the mechanical oscillator in a general fashion thus reads

$$
\omega\left(x_{0}+x\right)=\omega\left(x_{0}\right)+g \cdot x
$$

where we have introduced $g=\partial \omega / \partial x$ which is typically referred to as the optomechanical coupling coefficient. The Hamiltonian $\hat{H}$ of the coupled optomechanical system may then be written as a sum of the optical and mechanical subsystems' Hamiltonians $\hat{H}_{\text {opt }}$ and $\hat{H}_{\text {mech }}$, respectively, as well as the coupling Hamiltonian $\hat{H}_{\text {om }}$ [49]:

$$
\hat{H}=\hbar \omega \hat{a}^{\dagger} \hat{a}+i \hbar \sqrt{\kappa_{\mathrm{ex}}}\left(\hat{s} \hat{a}^{\dagger} \mathrm{e}^{-i \omega_{\mathrm{d}} t}-\hat{s}^{\dagger} \hat{a} \mathrm{e}^{i \omega_{\mathrm{d}} t}\right)+\hat{H}_{\kappa}+\hbar \Omega_{\mathrm{m}} \hat{b}^{\dagger} \hat{b}+\hat{H}_{\Gamma_{\mathrm{m}}}+\hbar g_{0}\left(\hat{b}^{\dagger}+\hat{b}\right) \hat{a}^{\dagger} \hat{a}
$$

The creation (annihilation) operators of the optical and mechanical modes are denoted as $\hat{a}^{\dagger}(\hat{a})$ and $\hat{b}^{\dagger}(\hat{b})$ with corresponding resonance frequencies $\omega\left(\omega \equiv \omega\left(x_{0}\right)\right)$ and $\Omega_{\mathrm{m}}$. The optical mode is coupled to an external port with rate $\kappa_{\mathrm{ex}}$, supplying a (laser) drive field $\hat{s} \mathrm{e}^{-i \omega_{\mathrm{d}} t}$ with mean photon flux $\left\langle\hat{s}^{\dagger} \hat{s}\right\rangle$ and angular frequency $\omega_{\mathrm{d}}$. The Hamiltonians $\hat{H}_{\Gamma_{\mathrm{m}}}$ and $\hat{H}_{k}$ describe the damping of the mechanical [58] and optical [59] modes with energy decay rates $\Gamma_{\mathrm{m}}$ and $\kappa$. The latter consists of coupling to the external port $\kappa_{\mathrm{ex}}$ and losses within the resonator $\kappa_{0}=\kappa-\kappa_{\mathrm{ex}}$. The interaction energy between optical and mechanical degree of freedom is determined by the vacuum optomechanical coupling rate

$$
g_{0} \equiv g \times x_{\text {zpf }}
$$

which is given by the product of optomechanical coupling coefficient $g$ and the zero-point fluctuations $x_{\mathrm{zpf}}=\sqrt{\hbar /\left(2 m_{\mathrm{eff}} \Omega_{\mathrm{m}}\right)}$ of the mechanical oscillator ( $m_{\text {eff }}$ denotes the oscillator's effective mass). The coupling Hamiltonian

$$
\hat{H}_{\mathrm{om}}=\hbar g_{0}\left(b^{\dagger}+b\right) a^{\dagger} a
$$

implements a mutual optomechanical coupling. It was introduced via the position dependent cavity resonance frequency (1). It also, however, represents a radiation pressure force $F$ that can be interpreted as

$$
\langle F\rangle=-\left\langle\frac{\partial \hat{H}}{\partial x}\right\rangle=-\hbar g\left\langle\hat{a}^{\dagger} \hat{a}\right\rangle
$$

leading to a per-photon force $-\hbar g$ on the mechanical oscillator. From the Hamiltonian (2) one can derive that this force gives rise to a static bistability [60], an optically induced rigidity [51,61] or dynamical backaction cooling/amplification characterized by the rate

$$
\Gamma_{\mathrm{dba}}=g_{0}^{2} \bar{a}^{2}\left(\frac{\kappa}{\kappa^{2} / 4+\left(\Delta+\Omega_{\mathrm{m}}\right)^{2}}-\frac{\kappa}{\kappa^{2} / 4+\left(\Delta-\Omega_{\mathrm{m}}\right)^{2}}\right)
$$

where $\bar{a}^{2} \equiv\left\langle\hat{a}^{\dagger} \hat{a}\right\rangle$, denotes the mean intracavity photon number.

Table 1 shows an overview of recently studied cavity optomechanical systems that realize the Hamiltonian (2). As can be seen, the corresponding system parameters vary over many orders of magnitude. A common feature of all studied systems is 
that the vacuum optomechanical coupling rate $g_{0}$ is at least three orders of magnitude smaller than the optical decay rate $\kappa$. For a few systems, however, $g_{0}$ can exceed the mechanical decay rate $\Gamma_{\mathrm{m}}$, in particular the near-field cavity optomechanical system presented here.

\section{Theoretical characterization of the near-field optomechanical coupling}

In this section, the evanescent optomechanical coupling between the optical modes of a toroid microresonator and a dielectric positioned in its evanescent near-field is examined theoretically. The whispering gallery modes supported by toroid microresonators exhibit an evanescent field such that the presence of a dielectric in this field should lead to a varying red shift of the optical resonance frequency depending on the distance to the dielectric. First, analytic expressions of the optomechanical coupling based on an approximation of the optical mode profile of toroid microresonators are given Second, these are compared to results of a finite element simulation which yields good agreement.

\subsection{Analytic optomechanical coupling}

For an optical mode $E^{(0)}$ in a dielectric structure characterized by $\epsilon^{(0)}(\vec{r})$ and a local perturbation $\epsilon^{(1)}(\vec{r})$ (induced by e.g. the presence of a dielectric nanomechanical oscillator in the evanescent field of a microresonator mode), first order perturbation theory yields the following fractional resonance frequency shift:

$$
\frac{\Delta \omega}{\omega}=-\frac{1}{2} \frac{\int \epsilon^{(1)}(\vec{r})\left|\vec{E}^{(0)}(\vec{r})\right|^{2} \mathrm{~d} \vec{r}}{\int \epsilon^{(0)}(\vec{r})\left|\vec{E}^{(0)}(\vec{r})\right|^{2} \mathrm{~d} \vec{r}}
$$

Using the optical resonator's mode volume $V$, Eq. (7) can be written as

$$
\frac{\Delta \omega}{\omega}=-\frac{1}{2} \frac{\int\left(\epsilon^{(1)}(\vec{r})-1\right)\left|\vec{E}^{(0)}(\vec{r})\right|^{2} \mathrm{~d} \vec{r}}{n^{2} V\left|E_{\max }\right|^{2}}
$$

Here, $\vec{E}_{\text {max }}$ denotes the maximum of the electric field $\max _{\vec{r}}\left|\vec{E}^{(0)}(\vec{r})\right|$ and $n$ is the refractive index of the optical resonator, which is assumed to be constant.

The fundamental optical modes of a toroid (major radius $R$ and minor radius $r$ ) can be approximated by

$$
E_{\zeta} \approx \begin{cases}E_{0} \exp \left[-\frac{z^{2}}{2 r_{z}^{2}}\right] J_{\ell}\left(T_{\ell 1} \frac{\rho}{\bar{R}}\right) \mathrm{e}^{i \ell \varphi}, & \rho<R \\ \frac{1}{P} E_{0} \exp \left[-\frac{z^{2}}{2 r_{z}^{2}}\right] J_{\ell}\left(T_{\ell 1} \frac{R}{\bar{R}}\right) \mathrm{e}^{i \ell \varphi} \mathrm{e}^{-\alpha(\rho-R)}, & \rho>R\end{cases}
$$

where $\bar{R}=R+\frac{P}{k_{0} \sqrt{n^{2}-1}}$ is an effective radius and $T_{\ell 1}$ denotes the first zero of the cylindrical Bessel function $J \ell^{1}$ The refractive index of the toroid is denoted by $n$ and $k_{0}$ is the (vacuum) wavenumber $k_{0}=2 \pi / \lambda$, where $\lambda$ denotes the (vacuum) wavelength. The modes can be approximately treated as TE-like modes, for which $P=1$ and $\zeta \approx z$ and TM-like modes for which $P=1 / n^{2}$ and $\zeta \approx \rho$ [62]. The mode width $r_{z}$ in $z$ direction, the evanescent decay length $\alpha^{-1}$ and the mode number $\ell$ are given by $r_{z} \approx R^{3 / 4} r^{1 / 4} \ell^{-1 / 2}$

$$
\begin{aligned}
& \alpha^{-1} \approx \frac{\lambda}{2 \pi \sqrt{n^{2}-1}} \\
& n k_{0} R \approx \sqrt{T_{\ell 1}^{2}+\ell \sqrt{R / r}}-P n / \sqrt{n^{2}-1}
\end{aligned}
$$

Moreover, we define $\xi=|E(\rho=R, z=0, \varphi)|^{2} /\left|E_{\max }\right|^{2}$ as the ratio of the field amplitude at the toroid's (vacuum) surface and the maximum field.

Now, a thin (rectangular) dielectric (thickness $t$, length $l_{y}$, width $l_{z}$ ) positioned tangentially at a distance $x_{0}$ from the toroid, as depicted in Fig. 3 (insert, left panel), is considered. Using Eq. (8), the optical frequency shift is thus given by:

$$
\Delta \omega\left(x_{0}\right)=-\frac{\omega}{2} \frac{\xi}{V}\left(n_{\text {diel }}^{2}-1\right) \int_{x=x_{0}}^{x_{0}+t} \int_{y=-l_{y} / 2}^{l_{y} / 2} \int_{z=-l_{z} / 2}^{l_{z} / 2} \mathrm{e}^{-\frac{z^{2}}{r_{z}^{2}}} \mathrm{e}^{-2 \alpha \sqrt{(x+R)^{2}+y^{2}}-R} \mathrm{~d} x \mathrm{~d} y \mathrm{~d} z
$$

The integral over the $z$-coordinate yields the sampling length in this direction ${ }^{2}$ :

\footnotetext{
1 For $\ell \gg 1$, as is the case here, these can be approximated by $T_{\ell 1}=\ell+1.8558 \ell^{1 / 3}+\mathcal{O}\left(\ell^{-1 / 3}\right)$.

2 The usual convention of defining the error function as $\operatorname{Erf}(x)=\frac{2}{\sqrt{\pi}} \int_{0}^{x} \mathrm{e}^{-t^{2}} \mathrm{~d} t$ is used.
} 

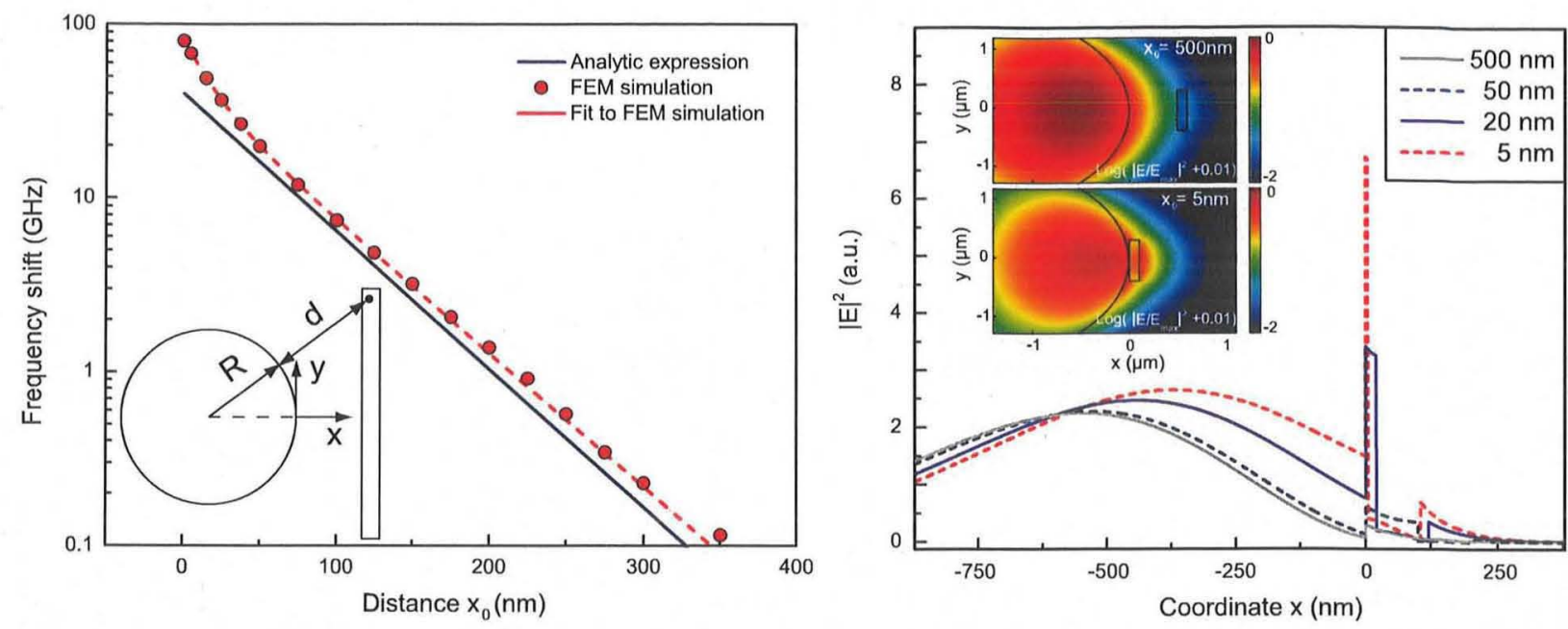

Fig. 3. Left panel: negative optical resonance frequency shift according to the analytic approximation (15) and FEM simulations as explained in the text for a TM-like mode, with typical parameters $R=16 \mu \mathrm{m}, r=1.7 \mu \mathrm{m}, \ell=171$ (corresponding to $\lambda \sim 800 \mathrm{~nm}$ ) and a nanomechanical string with crosssection $100 \mathrm{~nm} \times 700 \mathrm{~nm}$ (Fit model: sum of two exponentials). Insert: Typical geometry (top view) of circular toroid and rectangular dielectric. Right panel: Distortion of the profile of TM-like optical microtoroid mode induced by a silicon nitride nanomechanical oscillator in the evanescent field. The main panel shows the radial distribution of a TM mode for varying distances $x_{0}$ to the nanomechanical oscillator $(100 \mathrm{~nm} \times 700 \mathrm{~nm})$. The insert shows the corresponding field distribution for $x_{0}=500 \mathrm{~nm}$ and $x_{0}=5 \mathrm{~nm}$, respectively. For small $x_{0}$ a perturbation of the optical mode becomes evident, pulling the mode locally towards the vacuum. This leads to the deviation from first order perturbation theory, as described in the text.

$$
\bar{l}_{z}=\int_{z=-l_{z} / 2}^{l_{z} / 2} \mathrm{e}^{-\frac{z^{2}}{r_{z}^{2}}} \mathrm{~d} z=\sqrt{\pi} r_{z} \operatorname{Erf}\left(\frac{l_{z}}{2 r_{z}}\right)
$$

The integral over $x$ and $y$ can be approximated by two independent integrals such that the sampling length along the $y$-axis can be written as

$$
\bar{l}_{y}=\sqrt{\pi} \sqrt{R \alpha^{-1}} \approx \sqrt{\frac{R \lambda / 2}{\sqrt{n^{2}-1}}}
$$

using Eq. (11). The frequency shift is thus given by:

$$
\Delta \omega\left(x_{0}\right)=-\frac{\omega}{2} \frac{\xi}{V}\left(n_{\text {diel }}^{2}-1\right) \frac{\bar{l}_{z} \bar{l}_{y}}{2 \alpha}\left(1-\mathrm{e}^{-2 \alpha t}\right) \mathrm{e}^{-2 \alpha x_{0}}
$$

Using the first order expression for $\xi / V$ and keeping only terms to first order, one arrives at a coupling coefficient of

$$
g\left(x_{0}\right)=\frac{1}{2^{3 / 2} \pi} \frac{\omega}{R} \frac{n^{2}\left(n_{\text {diel }}^{2}-1\right)}{\left(n^{2}-1\right)^{5 / 4}} \sqrt{\frac{\lambda}{R}} \operatorname{Erf}\left(\frac{l_{z}}{2 r_{z}}\right)\left(1-\mathrm{e}^{-2 \alpha t}\right) \mathrm{e}^{-2 \alpha x_{0}}
$$

Interestingly, the near-field approach exhibits a stronger scaling of the optomechanical coupling with wavelength than the scattering force approach. In the latter case, the coupling $g$ is simply inversely proportional to wavelength, whereas here, the optomechanical coupling scales as $g \propto\left(1-\mathrm{e}^{-13.2 t / \lambda}\right) / \sqrt{\lambda}$ which for $t \ll \alpha^{-1}$ simplifies to $1 / \sqrt{\lambda^{3}}$.

The optomechanical coupling coefficients given by Eq. (16) are approximately $\times 20$ smaller than the intrinsic optomechanical coupling to the internal mechanical modes of the toroid microresonator $\omega / R[4,12,15,54,63]$, assuming $x_{0} \ll 2 / \alpha$ and typical parameters for a silicon nitride nanomechanical string $\left(l_{y} \times l_{z} \times t=30 \times 0.7 \times 0.1 \mu \mathrm{m}^{3}, n_{\text {diel }}=2\right)$. This loss in coupling coefficient is, however, overcompensated by typically more than $\times 100$ larger zero-point motion of the string's fundamental mode such that even larger vacuum optomechanical coupling rates $g_{0}$ are expected for evanescent near-field coupling.

\subsection{Comparison to finite element modelling results}

Finite element modelling (FEM) is applied to simulate the optomechanical coupling between a silica microtoroid and a dielectric in its evanescent field. The simulation assumes an axisymmetric geometry which holds true for the toroid itself, but not the nanomechanical element. To correct for this, the frequency shifts calculated in the axisymmetric simulation are re-scaled by the actual tangential sampling length $\bar{l}_{y}$ of the evanescent cavity field as derived in the previous section, cf. Eq. (14). 

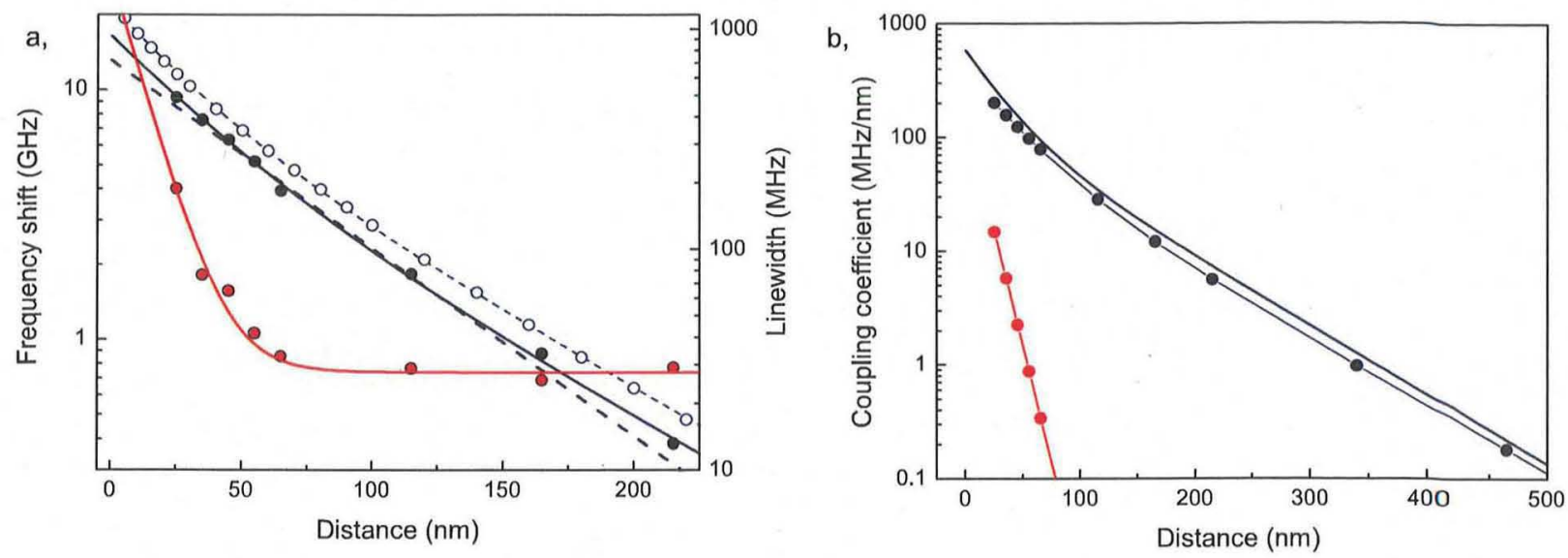

Fig. 4. Optomechanical coupling between toroid and silicon nitride nanomechanical string - theory and measurement. a, Negative optical frequency shift (full blue dots) and cavity linewidth (red dots) as a function of the distance to a nanomechanical string $\left(30 \times 0.3 \times 0.1 \mu \mathrm{m}^{3}\right)$ along with fits using a double (single) exponential decay for the frequency shift (linewidth). Empty dots: FEM simulation; dashed line: analytic approximation (Eq. (15)), both for TM-like mode. b, Red and blue dots represent the reactive and dispersive optomechanical coupling coefficients $\gamma$ and $g$, as given by the derivative of the exponential fits in a. Good agreement of the measured dispersive coupling with the FEM results (solid blue line) is found.

Fig. 3a shows the cavity resonance frequency shift according to Eq. (15) as well as calculated by FEM simulations for a TM optical mode and typical experimental parameters. A refractive index of $n_{\text {diel }}=2.0[64,65]$ is assumed ${ }^{3}$ for the silicon nitride string. Good agreement on a level of $15 \%$ is found between the analytic approximations of the resonance frequency shift and the results of the FEM simulation. Only for very small distances $x_{0}<50 \mathrm{~nm}$, the frequency shift evaluated by FEM shows a deviation from first order perturbation theory. This deviation is caused by the fact that for small distances the dielectric can significantly disturb the optical mode profile, pulling it slightly out of the silica structure and thus leading to larger frequency shifts, as shown in Fig. 3b. For small gaps $(\leqslant 50 \mathrm{~nm})$, the first order modifications of the optical mode profile caused by the dielectric mechanical oscillator cannot be neglected anymore and lead to a deviation from first order perturbation.

\section{Static measurement of the optomechanical coupling}

We will now turn to a careful experimental examination of the optomechanical coupling via the evanescent optical field. First the coupling to a single optical mode is analyzed, resulting in good agreement with the numerical values obtained in the previous section. Second, effects related to the simultaneous coupling to two different optical modes of similar optical frequency will be shown.

\subsection{Dispersive optomechanical coupling}

The simplest way to characterize the optomechanical coupling coefficient $g$ is based on static measurements of the cavity resonance frequency. To this end, the nanomechanical oscillator's distance $x_{0}$ to the cavity is gradually changed while a sweeping laser tracks the cavity resonance frequency $\omega\left(x_{0}\right)$. The position dependent optomechanical coupling coefficient $g\left(x_{0}\right)$ is then given by the derivative $\partial \omega / \partial x_{0}$ of the measured frequency shift.

Earlier measurements [28] allowed dispersive coupling coefficients $\mathrm{g} / 2 \pi$ up to $10 \mathrm{MHz} / \mathrm{nm}$ for silicon nanomechanical strings and membranes. No measurable reactive contribution was found [28]. Residual vibrations between the nanomechanical oscillator and microresonator chips within the experimental apparatus practically limited the gaps which allowed stable measurements to an estimated value of $x_{0} \sim 100 \mathrm{~nm}$ in this first series of experiments. Subsequently, efforts were made to increase the accessible optomechanical coupling coefficients. First, the vibration isolation of the experimental chamber was significantly improved. Moreover, the experimental parameters according to Eqs. (16) and (11), $g \propto\left(1-\mathrm{e}^{-13.2 t / \lambda}\right) / \sqrt{\lambda R^{3}}$, were optimized. Working at shorter wavelength by moving from the telecommunication wavelength band to the range of a titanium-sapphire laser $(\lambda \sim 800 \mathrm{~nm})$ enhanced the attainable coupling by a factor $\sim 2$. The fabrication process of the toroid near-field sensors was optimized to allow for smaller cavity radii, further increasing the coupling. High-finesse cavities with radii down to $16 \mu \mathrm{m}$ and a finesse $\sim 10^{6}$ were achieved, similar to regular toroid microresonators [69].

Fig. 4a shows the measured frequency shift induced by a narrow nanomechanical string $\left(30 \times 0.3 \times 0.1 \mu \mathrm{m}^{3}\right)$ for a resonance at $838 \mathrm{~nm}$ wavelength of a $18.4 \mu \mathrm{m}$-radius cavity along with the shift expected from both FEM simulations and

\footnotetext{
3 The refractive index depends on the silicon concentration of the silicon nitride. An error of \pm 0.1 is estimated [64,66], larger than the index variation $\sim 0.05$ as a function of wavelength between $600 \mathrm{~nm}$ and $1600 \mathrm{~nm}[67,68]$.
} 

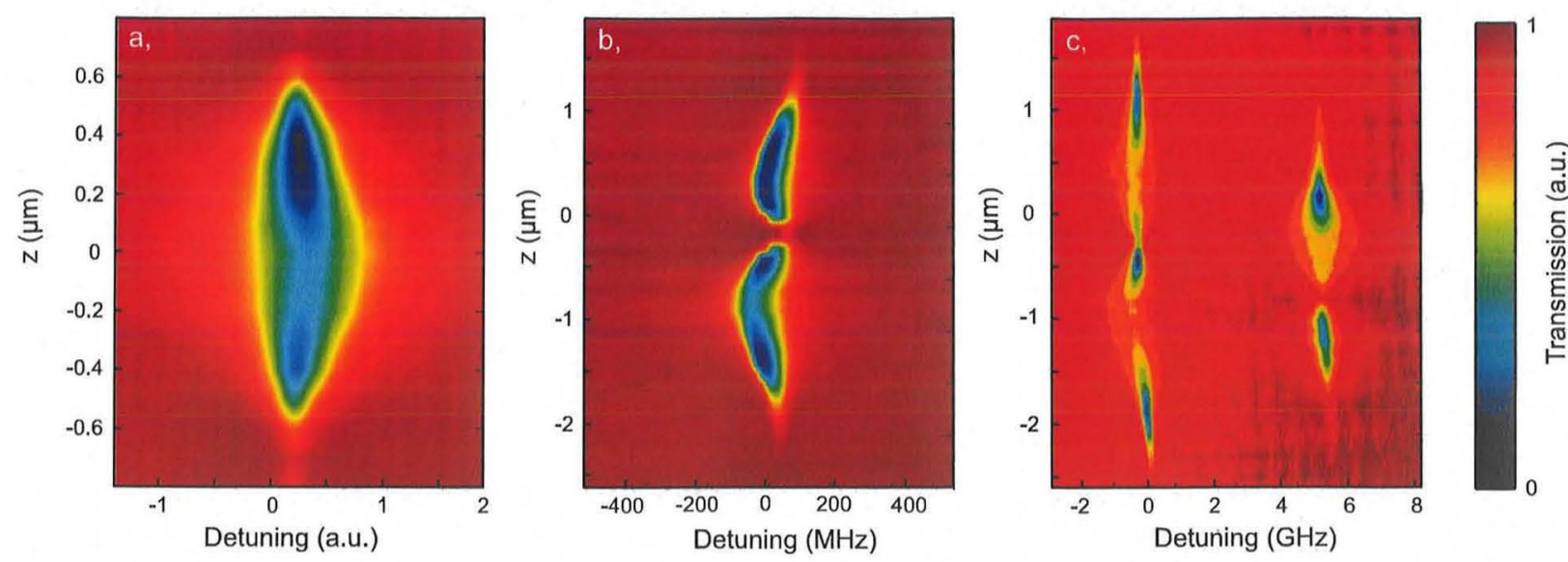

Fig. 5. Spatial profile of optical toroid microresonator modes as inferred by the transmission of the tapered optical fibre at varying distance. Fundamental (a, $|m|=\ell$ ), second order (b, $|m|=\ell-1)$, second and third order $(c,|m|=\ell-2)$ modes can be distinguished.

the analytic approximation (see Eq. (15)). A maximum frequency shift of $9 \mathrm{GHz}$ is measured and good agreement with both FEM simulation and analytic model is found, assuming a closest distance of $25 \mathrm{~nm}$. For very small gaps $x_{0}<50 \mathrm{~nm}$ the measured frequency shift deviates from the uniform exponential behaviour and follows a steeper slope, in agreement with the FEM simulation. This is attributed to the deviation from first order perturbation, as described in Section 3.2. Another important aspect is that given the increased dispersive coupling, now for small $x_{0}$ also the cavity linewidth considerably increases, as shown in Fig. 4a. This effect cannot be explained by absorption of photons in silicon nitride. Based on an upper bound for the ratio of imaginary to real part of the refractive index (the complex refractive of high stress SiN was measured to be $\lesssim 0.6 \times 10^{-5}$ at a wavelength of $935 \mathrm{~nm}$ in Ref. [70]), absorption should, in the case of Fig. 4a, lead to an increase of the linewidth by $<1 \mathrm{MHz}$, two orders of magnitude smaller than the observed value. The increased optical losses are rather attributed to increased surface scattering at both silicon nitride and silica surfaces since for small gaps the optical mode is considerably pulled towards these, cf. Fig. $3 \mathrm{~b}$.

Fig. 4b shows the dispersive and reactive coupling coefficients derived from the data in panel a. For all $x_{0}$, the dispersive contribution by far dominates the interaction. The measured dispersive coupling coefficients show very good agreement with the coefficients obtained from FEM simulations reaching values $g / 2 \pi>200 \mathrm{MHz} / \mathrm{nm}$ which corresponds to a more than one order of magnitude improvement compared to the initial values [28], attributed to shorter wavelength, smaller cavity radius and improved stability, i.e. smaller $x_{0}$. The reactive coupling $\gamma=\partial \kappa / \partial x$, in turn, reaches values up to $\gamma / 2 \pi \leqslant 13 \mathrm{MHz} / \mathrm{nm}$.

The largest dispersive coupling was measured using wider nanostring oscillators with a cross-section of $700 \times 100 \mathrm{~nm}^{2}$ reaching a value of $g / 2 \pi=290 \mathrm{MHz} / \mathrm{nm}$ which corresponds to a vacuum optomechanical coupling rate of $g_{0} / 2 \pi \sim 5 \mathrm{kHz}$ (with the zero-point fluctuations of this particular string given by $x_{\mathrm{zpf}} \sim 18 \mathrm{fm}$, cf. Section 5 ).

\subsection{Simultaneous coupling to several optical modes}

Due to the rich mode spectrum of toroid microresonators, situations might arise where two or more optical resonances closely spaced in frequency couple to the nanomechanical oscillator simultaneously.

Fig. 5 shows the measured vertical optical mode profiles of several optical modes. These are acquired by measuring the transmission of the tapered fibre while scanning both its position along the $z$-axis (the distance to the toroid is kept fixed) and the laser frequency. Fig. $5 \mathrm{a}$ shows a fundamental optical mode characterized by a single lobe along the $z$-coordinate. Figs. $5 b$ and $5 c$, however, show higher order optical modes with two and three lobes along the $z$-coordinate. Obviously, the coupling of a nanomechanical string to an optical mode doublet, as shown in Fig. 5c, strongly depends on its position along the $z$-axis.

Figs. 6a-6b show a situation where a nanomechanical string is coupled to an optical mode doublet. It is positioned close to a maximum (minimum) of the higher (lower) frequency mode. As the nanostring is approached to the toroid the higher frequency mode is exponentially shifted towards lower frequencies whereas the lower frequency mode is not affected. As the frequencies of both optical modes approach each other, the scattering induced by the nanomechanical oscillator strongly couples both modes, leading to an avoided crossing. During the avoided crossing both optical modes also switch their respective optical linewidth, as expected from a simple coupled oscillator model. It is noted that the corresponding coupling frequency is as large as $\sim 3 \mathrm{GHz}$, much larger than in a previously studied microsphere system [71] which is attributed to a Purcell enhancement of the scattering [71,72] into the adjacent high $Q$ optical modes $\left(Q \sim 5 \times 10^{5}\right.$ and $\left.Q \sim 3 \times 10^{7}\right)$. For the closest distance $x_{0} \sim 25 \mathrm{~nm}$, the higher frequency optical mode is shifted by $18 \mathrm{GHz}$ and a dispersive coupling coefficient of $g / 2 \pi=270 \mathrm{MHz} / \mathrm{nm}$ is achieved. If the coupling between both optical modes is weaker than one of the optical mode's intrinsic decay rates, a Fano-type interference is observed as both modes are tuned into resonance with each other. This situation is depicted in Fig. $6 \mathrm{c}$ where a very broad resonance (linewidth $\sim 5 \mathrm{GHz}$ ) is used. 

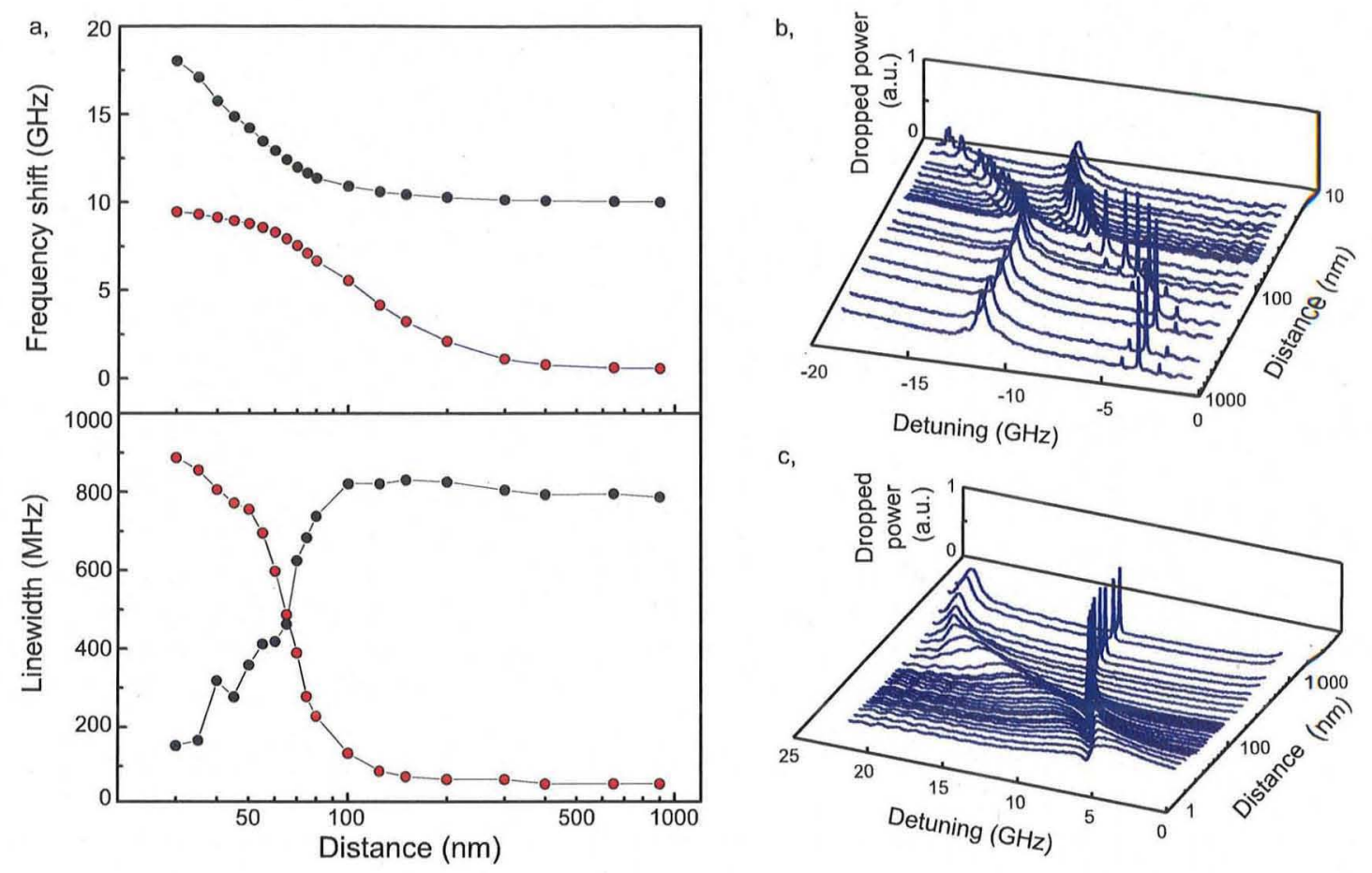

Fig. 6. Avoided crossing and Fano-type interference of optical microresonator modes induced by dielectric nanostrings. a, Negative optical resonance frequency shift (top) and linewidth (bottom) of two adjacent optical WGM microtoroid modes as a function of the distance to a nanomechanical silicon nitride string. For large distances only the higher frequency mode is coupled to the nanomechanical oscillator. Correspondingly, it is pulled towards the lower frequency mode as the nanomechanical string is approached. The dielectric nanomechanical oscillator distorts and couples both modes to each other via scattering which gives rise to an avoided crossing. b shows the corresponding cavity absorption spectra. c, Fano-like interference of optical microresonator modes. The situation is similar to b. Here, however, the higher frequency mode has a broad linewidth (broader than the coupling rate between both modes). Thus, it effectively acts as a continuum leading to a Fano-type interference.

\section{Transduction and actuation of nanomechanical motion}

With the previously characterized static optomechanical coupling coefficients which were obtained by displacing the nanomechanical oscillator as a whole, we now turn to the intrinsic mechanical modes of the employed nanomechanical oscillators. To this end, the Brownian motion of the nanomechanical oscillators positioned at a fixed distance $x_{0}$ to the microresonator will be examined. First, however, the notion of a mechanical oscillator's effective mass which is crucial for correctly scaling the optomechanical interaction is introduced.

\subsection{Effective mass of the nanomechanical oscillators}

In Section 4.1 the optomechanical coupling was defined as the static optical resonance frequency shift induced by an infinitesimal change in oscillator position $x_{0}, \Delta \omega \equiv \Delta x_{0} \cdot g$. Here, we will consider the cavity frequency noise $S_{\omega}[\Omega]$ induced by the Brownian position fluctuations $S_{x}[\Omega]$ of a particular mechanical mode of the nanomechanical oscillator $S_{\omega}[\Omega]=$ $g_{\text {dyn }} \cdot S_{X}[\Omega]$. The cavity frequency noise spectrum $S_{\omega}$ can be measured in absolute terms [73]. The quantities on the right hand side of the above equation are, however, a priori not that well defined. Typically, the convention of matching static and dynamic coupling coefficients $g_{\text {dyn }}\left(x_{0}\right) \equiv g\left(x_{0}\right)$ has been pursued in the optomechanical literature (which leads to the well-known $g(l)=\omega / l$ for a Fabry-Perot cavity of length $l$ ). This convention necessitates to carefully define the actually measured effective coordinate of each mechanical oscillator mode, taking into account the spatial overlap of the optical and mechanical mode shape used for measurement. Moreover, this convention requires the notion of an effective mass [74] for each mechanical oscillator mode in order to fulfill the equipartition theorem. Following a different convention, one could e.g. also absorb the overlap between optical and mechanical modes into an effective optomechanical coupling which would then lead to different optomechanical coupling for each individual mechanical mode and in particular different static and dynamic coupling coefficients. Then, in turn, the notion of constant motional mass for all mechanical modes could be adopted.

Here, we follow the former, more frequently used convention as then both static and dynamic coupling coefficients are identical for all mechanical modes. In the following, the effective coordinates and corresponding effective masses for a nanomechanical string positioned horizontally in the evanescent optical microresonator field will be derived. A simple 
method for characterizing the optomechanical coupling via direct measurements of the vacuum optomechanical coupling rate $g_{0}$ that avoids having to introduce effective masses or effective coupling coefficients is reported in Ref. [73].

The $j$-th eigenfrequency of a doubly clamped rectangular string of length $L$, Young's modulus $Y$ and cross-sectional inertia $I=w t^{3} / 12$ (the thickness $t$ is understood along the axis of oscillation and the width $w$ corresponds to the lateral dimension) is given by [75]:

$$
\Omega_{\mathrm{m}}^{j} /(2 \pi)=\frac{j^{2} \pi}{2 L^{2}} \sqrt{\frac{Y I}{\rho A}} \sqrt{1+\frac{A S L^{2}}{j^{2} Y I \pi^{2}}}
$$

In the limit of large internal tensile stress $\left(S \gg(j \pi t / L)^{2} Y / 12\right)$, the mode patterns of a nanomechanical string extending from $y=0$ to $y=L$ are given by

$$
u_{j}(y)=u_{j, 0} \sin \left(\frac{j \pi}{L} y\right)
$$

Each mechanical mode fulfills the equipartition theorem:

$$
\frac{1}{2} m \Omega_{\mathrm{m}}^{2}\left\langle u_{j}^{2}\right\rangle=k_{B} T
$$

where $m=\rho t w L$ denotes the oscillator's physical mass and $\left\langle u_{j}^{2}\right\rangle=1 / L \int_{L} u_{j}(y)^{2} \mathrm{~d} y$ is the mean squared displacement amplitude averaged along the string's length. ${ }^{4}$

In general, however, the measured coordinate deviates from $\left\langle u_{j}^{2}\right\rangle$ since it is weighted by the geometry of the measurement apparatus. For the presented system the measured coordinate is given by the spatial distribution of the normalized optical mode profile $v_{0}(y)\left(\int_{-\infty}^{\infty} v_{0}(y)^{2} \mathrm{~d} y=1\right)$, sampling the nanomechanical string. This results in a normalized squared displacement of

$$
\left\langle u_{j}\right\rangle_{v_{0}^{2}}^{2}=\left(\int_{L} u_{j}(y) v_{0}(y)^{2} \mathrm{~d} y\right)^{2}
$$

which generally does not equal $\left\langle u_{j}\right\rangle^{2}$. In order to maintain equipartition for the actually measured coordinate the oscillator's mass correspondingly has to be adjusted. This leads to an individual effective mass $m_{\text {eff }}$ for each mechanical mode. Using Eqs. (18), (19) and the approximate expression of the toroid field as obtained in the previous section $\left(v_{0}^{2}(y)=\sqrt{\frac{\alpha}{\pi R}} e^{-\alpha\left(y-y_{0}\right)^{2} / R}\right.$, with the field maximum along the string residing at $\left.y=y_{0}\right)$ one obtains the following expression for the effective mass $m_{\mathrm{eff}}^{j}$ of the $j$ th mode:

$$
m_{\mathrm{eff}}^{j}=\frac{m}{2}\left(\int_{0}^{1} \beta \sin (j \pi u) \operatorname{Exp}\left[-\pi \beta^{2}\left(u-\frac{y_{0}}{L}\right)^{2}\right] \mathrm{d} u\right)^{-2}
$$

where $\beta^{2}=\frac{L^{2} \alpha}{\pi R}=L / \bar{l}_{y}$ reflects the string's length $L$ weighted by the transverse optical sampling length $\vec{l}_{y}$.

Typical experimental parameters yield $\beta \gg 1$. For the fundamental mechanical modes $(j=1)$ and symmetric probing around the string's centre $\left(y_{0}=L / 2\right)$, the integrand in the above expression thus approximately represents a normalized Gaussian (centered at $u=1 / 2$ with a width $\ll 1$ ). Correspondingly, values close to 1 are found for the integral. Thus, the effective mass of the fundamental modes is typically given by half the oscillator's physical mass, i.e. $m_{\text {eff }} \approx m / 2$. It is noted that the corresponding identity of static and dynamic coupling coefficients was demonstrated in Ref. [28]. For higher order mechanical modes whose motion is more localized, however, the deviation from $m_{\text {eff }}^{j}=m / 2$ can be large.

\subsection{Mechanical modes of silicon nitride nanomechanical strings}

The dispersive optomechanical coupling $g$ transduces the Brownian motion of the nanomechanical oscillators into cavity frequency noise $S_{\omega}[\Omega]$ via:

$$
S_{\omega}[\Omega]=g^{2} \times S_{x}[\Omega]
$$

When the cavity is probed by a laser field using a tapered fibre, the Brownian motion $S_{x}$ of the nanomechanical oscillator will thus be imprinted onto phase and amplitude fluctuations of the field transmitted through the tapered optical fiber coupled to microresonator and nanomechanical oscillator (cf. Fig. 1). Spectral analysis of the corresponding amplitude

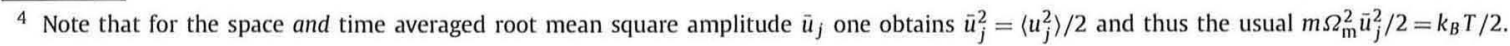



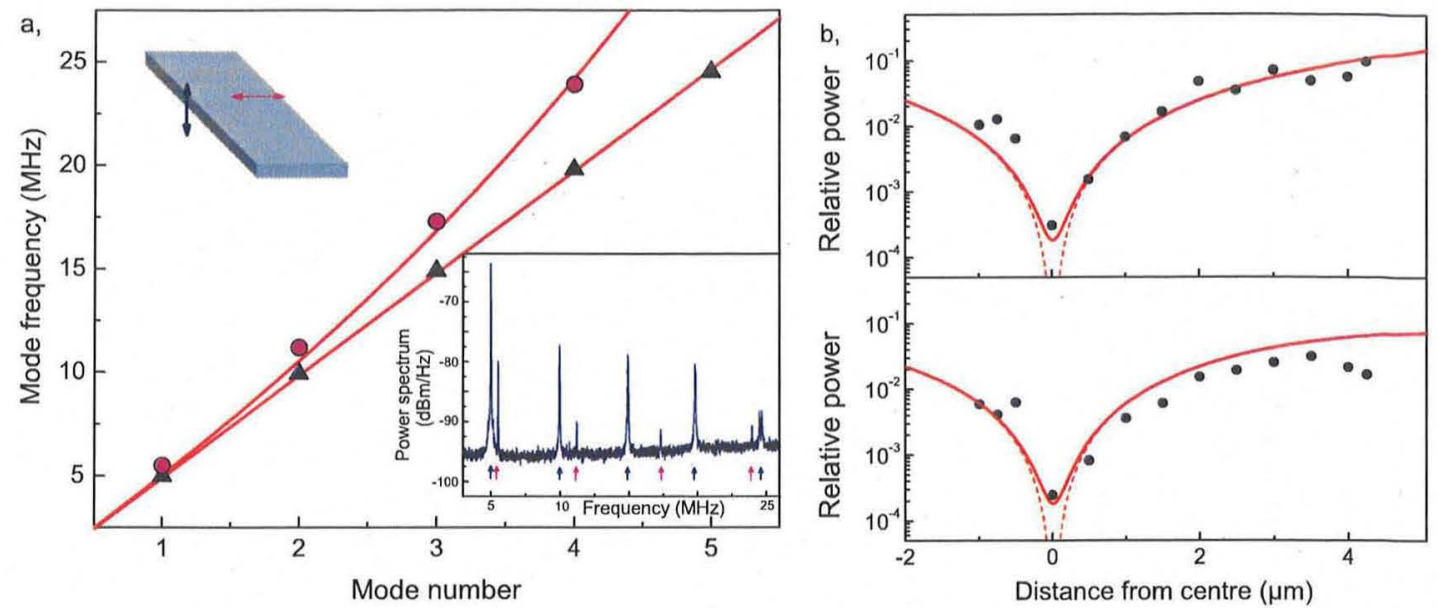

Fig. 7. Modes of a $40 \times 0.7 \times 0.1 \mu \mathrm{m}^{3}$ nanomechanical string. a, Out-of-plane (triangles) and in-plane mechanical modes (circles). The out-of-plane mode family follows a harmonic frequency ladder whereas the in-plane mode family increases faster in frequency owing to the larger in-plane cross-sectional inertia (insert: corresponding spectrum). Displacing the toroid along the string's axis allows recording the nanomechanical string's spatial mode patterns, as shown in b: peak squared displacement of the second (upper panel) and fourth (lower panel) out-of-plane mode normalized to the fundamental mode. Good agreement with the values expected from Eq. (18) (dashed lines) is found, when the finite sampling length of the microresonator $\bar{l}_{y}$ (cf. Eq. (14)) is taken into account (full lines).

or phase fluctuations then allows retrieving the Brownian noise spectrum of the nanomechanical oscillator, as shown in Fig. 7a (insert). This allows measuring nanomechanical motion with an imprecision below the level of the standard quantum limit [29].

\subsection{Frequency dispersion and mode patterns of nanomechanical oscillators}

In this section the properties of the employed nanomechanical strings will be characterized. Intrinsic mechanical quality factors ranging from $10^{4}$ to $>10^{5}$ are found (for frequencies of $5 \mathrm{MHz}$ to $25 \mathrm{MHz}$ ) in vacuum (pressure $<10^{-5} \mathrm{mbar}$ ). These are similar to the values reported elsewhere for nanostrings [65]. The measured mechanical quality factors are most likely limited by two level systems within the amorphous silicon nitride $[76,77]$. In the following, we will focus on the mode structure of nanomechanical strings.

Fig. 7a shows the nine lowest order eigenmodes of a $40 \times 0.7 \times .1 \mu \mathrm{m}^{3}$ nanomechanical string. The out-of-plane modes follow a linear dependence on mode number, here up to the fifth mode at $25 \mathrm{MHz}$ since their motion is dominated by the internal tensile stress, i.e. $S \gg(j \pi t / L)^{2} Y / 12$ (cf. Eq. (17)). The spatial displacement patterns of these modes can be measured by displacing the toroid microresonator with respect to the centre of the nanostring. Fig. 7b shows the squared oscillation amplitude of the second and fourth out-of-plane modes normalized to the fundamental out-of-plane mode. The measured amplitudes are in good agreement with the theoretical expectation taking into account the transverse optical sampling length $\bar{l}_{y}$ (see Eq. (14)). In fact, minimizing the signal of even eigenmodes constitutes a simple way to find the centre of the nanostrings and thus optimize the transduction of the fundamental mode. The measured out-of-plane modes allow inferring the ratio $S / \rho=(1.6 \pm 0.1) \times 10^{5} \mathrm{~m}^{2} / \mathrm{s}^{2}$. For LPCV deposited high-stress silicon-nitride a density of $(3 \pm$ $0.5) \times 10^{3} \mathrm{~kg} / \mathrm{m}^{3}$ is expected, see Ref. [65] and references therein. Thus, the resulting tensile stress in this particular sample is given by $S=(0.5 \pm 0.1) \mathrm{GPa}$. Values up to $S=0.8 \mathrm{GPa}$ were, however, obtained using other samples. The measurement is moreover also sensitive to in-plane modes of the string (a higher order optical mode is used for this measurement). Owing to the high aspect-ratio of the nanomechanical oscillators, these exhibit considerably different frequencies and in particular do not follow linear dispersion, as shown in Fig. 7a. This is due to the increased value of the cross-sectional inertia (by a factor $(w / t)^{2}$ ) for modes oscillating along the longer axis of the string. With this additional mode family, the Young's modulus of the employed strings can be calculated. Using the above value for density and stress in order to fit expression (17) to this mode family (see Fig. 7a), one obtains a Young's modulus of $Y=(55 \pm 9)$ GPa which is lower than the value measured in Ref. [65] for higher stress $(S \approx 1.2 \mathrm{GPa}$ ) LPCVD silicon nitride ( $Y \approx 200 \mathrm{GPa}$ ).

\section{Dynamical backaction cooling and amplification using a single optical mode}

In this section dynamical backaction effects using a single optical mode are presented. Exceptionally low threshold power levels $(P<1 \mu \mathrm{W})$ for the onset of regenerative nanomechanical oscillations are found. Moreover, the feasibility of cooling nanomechanical motion by radiation pressure is shown.

Pumping the optomechanical system with a detuned laser alters the damping rate of the mechanical oscillator to $\Gamma_{\text {eff }}=$ $\Gamma_{\mathrm{m}}+\Gamma_{\mathrm{dba}}$ where $\Gamma_{\mathrm{dba}}$ is given by Eq. (6). Pumping the cavity with a blue detuned laser beam thereby leads to antidamping of the employed nanomechanical string which can lead to maser/laser-like amplification of mechanical motion 

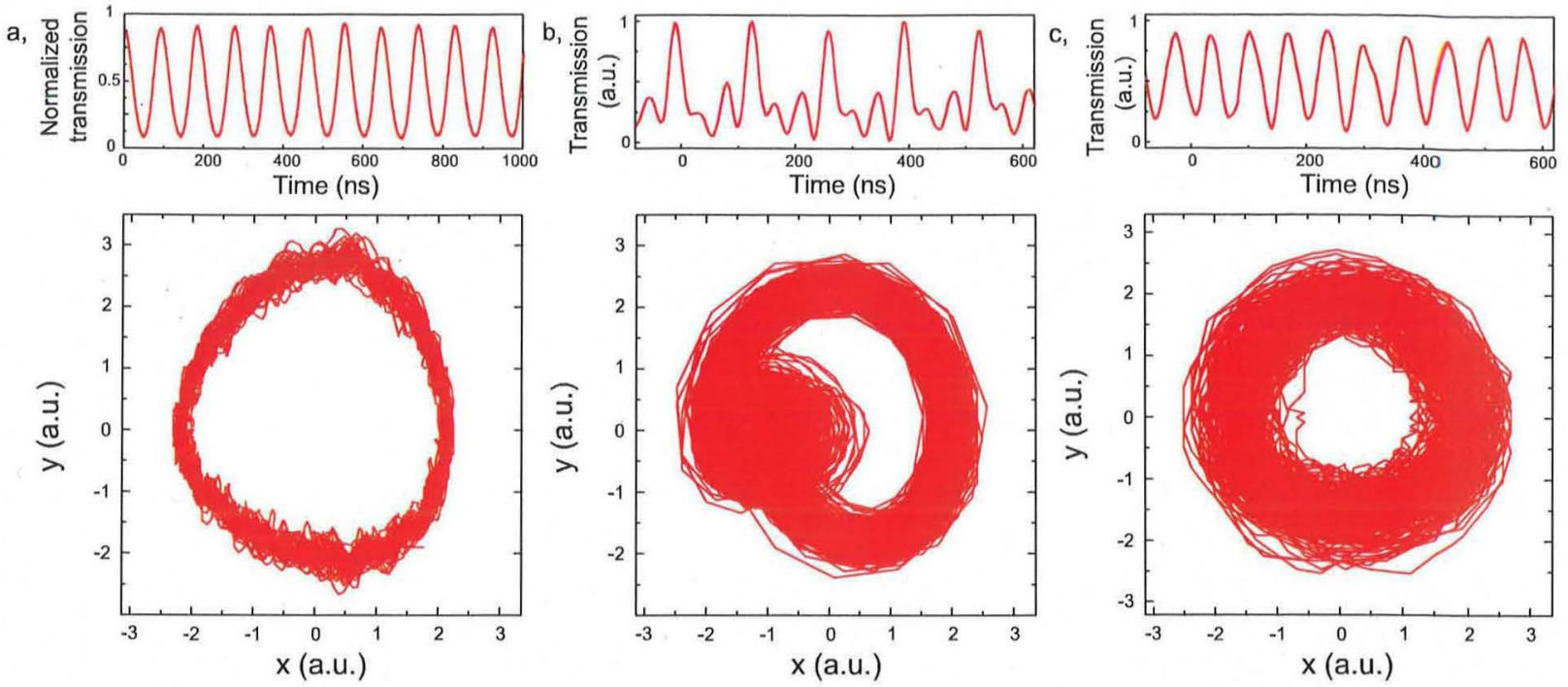

Fig. 8. Temporal behaviour of self-sustained oscillations. The coherent nanomechanical oscillations cause near-unity modulation of the cavity transmission (a, $\Omega_{\mathrm{m}} / 2 \pi=10.8 \mathrm{MHz}, Q_{\mathrm{m}}=70000, m_{\text {eff }}=3.6 \times 10^{-15} \mathrm{~kg}$ ). Here, an input power of $\sim 10 \mu \mathrm{W}$ and a coupling coefficient of $\mathrm{g} / 2 \pi \sim 5 \mathrm{MHz} / \mathrm{nm}$ is used. For higher coupling power the transmission through the optical resonator becomes nonlinear as depicted in b and period doubling can be observed, $\mathrm{c}$. These data were obtained for a $7.8 \mathrm{MHz}$ nanomechanical string $(P \sim 5 \mu \mathrm{W}, \mathrm{g} \sim 25 \mathrm{MHz} / \mathrm{nm})$. The oscillations in the nonlinear regime are, however, less stable in amplitude as can been seen in the corresponding phase diagrams.

once the (negative) dynamical backaction rate $\Gamma_{\mathrm{dba}}$ overcomes the intrinsic damping rate of the mechanical oscillator, i.e. at $\left|\Gamma_{\mathrm{dba}}\right| \geqslant \Gamma_{\mathrm{m}}$. The first experimental observation of the radiation pressure parametric instability was reported in 2005 [4]. The near-field platform allowed to transfer this effect to the nanoscale [28]. In Fig. 8a, the transmission of the microresonator is shown both in time domain and in a phase space representation. A near unity modulation of the cavity transmission is caused by the coherent oscillations of the nanomechanical oscillator in the regime of the parametric oscillation instability.

The observation of radiation pressure induced coherent oscillations of Ref. [28] constituted the first report of dynamical backaction amplification or cooling of nanomechanical motion using radiation pressure (in contrast to thermal effects $[36,78])$, and in particular using optical gradient or dipole forces. A radiation pressure induced optical spring effect within a nano-optomechanical system has been reported in Ref. [36] using a photonic crystal cavity. Dynamical backaction cooling or amplification of nanomechanical oscillators had earlier on, however, been achieved only using single electron transistors [32] or microwave fields [17] which require cryogenic operation and, owing to more than four orders of magnitude longer wavelength, show much lower coupling coefficients. Meanwhile, also optomechanical crystals allowed the observation of backaction amplification [46].

As the coupling coefficient $g$ (or equivalently $\sqrt{P}$ ) is increased in the presented near-field system, the mechanical oscillations may shift the cavity resonance frequency by more than its linewidth such that the transmission of the optomechanical system becomes nonlinear. This is shown in Figs. 8b-8c. These oscillations are achieved with minute input powers of only $P \sim 5 \mu \mathrm{W}$ which is about three orders of magnitude less than the power levels used in [5] where similar behaviour is reported for a micromechanical oscillator.

Fig. 9 shows the measured threshold power for the parametric instability as a function of the employed optomechanical coupling. A remarkably low value of $P_{\text {th }}=400 \mathrm{nW}$ is found for a nanomechanical string $\left(\Omega_{\mathrm{m}} / 2 \pi=8.4 \mathrm{MHz}, Q_{\mathrm{m}}=60000\right.$, $m_{\text {eff }}=3 \times 10^{-15} \mathrm{~kg}$ ) using a coupling coefficient of $\mathrm{g} / 2 \pi=45 \mathrm{MHz} / \mathrm{nm}$.

The radiofrequency signal caused by the mechanical oscillator in the parametric oscillation regime has been proposed to serve as a photonic clock [8] motivated by its ultra-narrow linewidth [79]. As such, low-power, radiation pressure driven nanomechanical oscillators might be able to serve as on-chip frequency references replacing the commonly used bulkier quartz crystals. Such an application, however, requires a thorough study and minimization of the signal's phase noise [6-8] to reach the quality of a quartz oscillator.

The opposite regime, namely dynamical backaction cooling, is also feasible, yielding a tool for radiation pressure cooling nanomechanical motion. Fig. $9 \mathrm{~b}$ shows the spectra recorded with a nanomechanical string $\left(\Omega_{\mathrm{m}} / 2 \pi=10.8 \mathrm{MHz}\right.$, $\left.Q_{\mathrm{m}}=50000, m_{\mathrm{eff}}=3.2 \times 10^{-15} \mathrm{~kg}, \kappa / 2 \pi=8 \mathrm{MHz}, P=14 \mu \mathrm{W}, \mathrm{g} / 2 \pi=4 \mathrm{MHz} / \mathrm{nm}\right)$ with both a positively and negatively detuned laser. The former $(\Delta / 2 \pi \sim 10 \mathrm{MHz})$ leads to linewidth narrowing, and a corresponding effective temperature increase to $T_{\text {eff }}=570 \mathrm{~K}$. The latter $(\Delta / 2 \pi \sim-10 \mathrm{MHz})$ increases the oscillator's intrinsic linewidth $\Gamma_{\mathrm{m}} / 2 \pi=220 \mathrm{~Hz}$ via dynamical backaction to $\Gamma_{\text {eff }}=940 \mathrm{~Hz}$ which leads to cooling by a factor $\Gamma_{\text {eff }} / \Gamma_{\mathrm{m}} \sim 4.3$, i.e. a temperature of $70 \mathrm{~K}^{5}$

\footnotetext{
5 The effective temperature is in both cases inferred by the oscillator linewidth.
} 

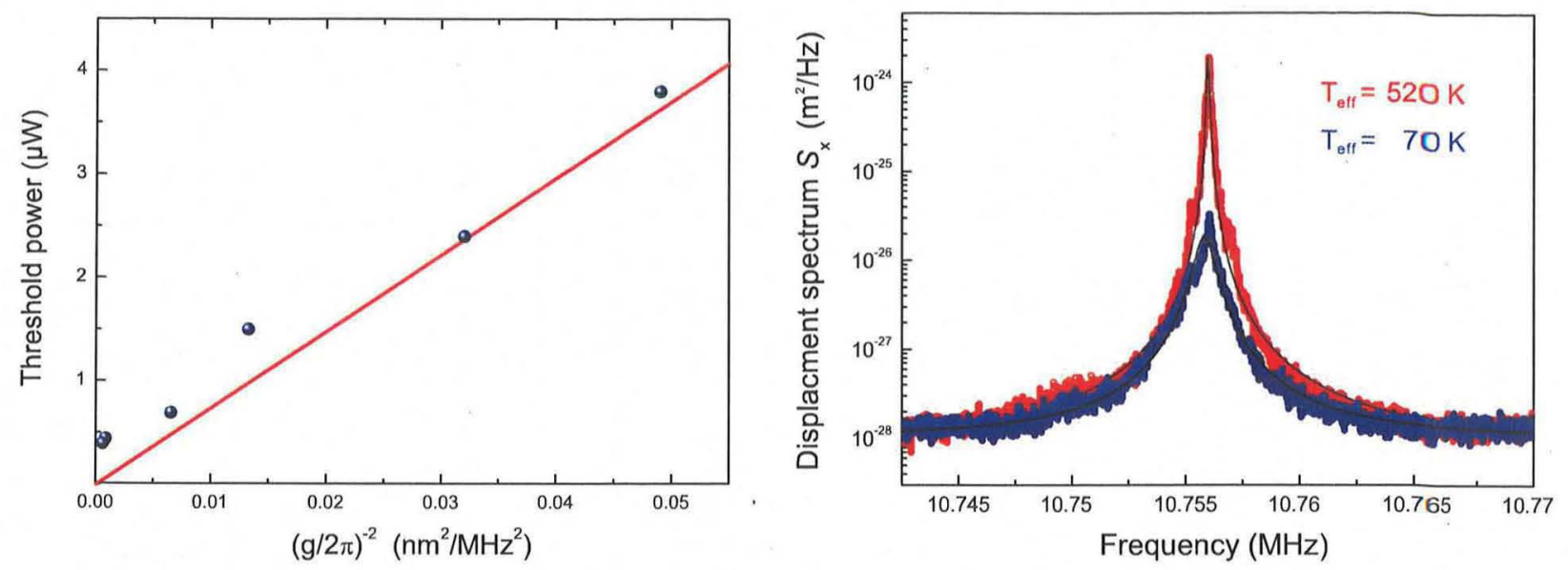

Fig. 9. Dynamical backaction amplification and cooling. a, Threshold power for the onset of self-sustained mechanical oscillations. $P_{\text {th }}$ is shown as a function of the optomechanical coupling $g$. The lowest threshold power obtained is $400 \mathrm{nW}$. The line is the theoretical expectation from Eq. (6) with no fit parameters. b, Dynamical backaction cooling and heating of a nanomechanical oscillator. The blue (red) trace shows the (single-sided) motional spectrum of a nanostring (properties as described in the text) measured with a negative (positive) detuning of $|\Delta| \sim 10 \mathrm{MHz}$. Correspondingly its effective temperature is reduced (increased) to $70 \mathrm{~K}(520 \mathrm{~K})$

Recent experiments with micromechanical oscillators have shown much stronger backaction cooling, reaching temperatures of $\sim 5 \mathrm{~K}[10-12,15]$ (i.e. cooling by $\times 10^{2}$ ) or even in the low mK-range [16] (i.e. cooling by $\times 10^{4}$ ), starting from room temperature. Cryogenic implementations of micro-optomechanical systems have, moreover, reached occupation numbers of only ten or a few tens of quanta $[19,20,52,63]$ with a backaction cooling contribution within $10-10^{3}$. Radiation pressure backaction cooling of nanomechanical oscillators, however, has previously only been shown using superconducting microwave systems and cooling factors of $\times 5$ [17] and $\times 100$ [21], limited by thermal effects, have been realized.

With the parameters $\kappa / \Omega_{\mathrm{m}}=0.74<\sqrt{12}$ as used here, in principle cooling to a final occupation below $1 / 2$ is possible $[13,14]$. In order to achieve this goal, considerably larger cooling factors would, however, have to be achieved. This corresponds to using larger optomechanical coupling, larger input power or higher mechanical $Q$. A $\times 10$ larger coupling coefficient as well as two times higher mechanical $Q$ are in principle easily accessible. This would hypothetically then require a still realistic but nevertheless very large input power, i.e. $P=9.4 \mathrm{~mW}$, to reach an occupation below unity from room temperature. The strong thermal bistability of toroid microresonators [80-82] would certainly render it very difficult to reliably lock such a high power beam to the red wing of the optical resonance at room temperature. At temperatures accessible using He-4 cryostats $(T=1.6 \mathrm{~K}$ ), however, cooling below unity should be feasible with an estimated (based on the above parameters) input power of only $P=50 \mu \mathrm{W}$, where the expected increase in mechanical $Q$ of silicon nitride based resonators at cryogenic temperatures [76] is not even taken into account.

\section{Dual optical mode cooling and amplification}

So far, we have only considered a single electromagnetic mode coupled to a single mechanical mode, according to Eq. (2). In the literature, however, also the interaction of several optical modes with a mechanical oscillator has been studied both theoretically $[48,83,84]$ and experimentally $[85,86]$. Such an interaction can considerably enhance both dynamical backaction and transduction of motion.

In general, the effective optomechanical coupling rate $g_{0} \bar{a}^{2}[18]$ is increased by using high quality factor optical resonances, i.e. by small $\kappa$. In the case $\kappa \ll \Omega_{\mathrm{m}}$ the optical resonator's response, however, becomes too slow to follow the mechanical oscillator's dynamics which cancels the benefit of high optical $Q$. Thus, the system's performance, as described e.g. by the power needed to reach the SQL $\left(P_{\mathrm{SOL}}\right)$, or equivalently the threshold power to reach the parametric instability [29], saturates to a value independent of the cavity bandwidth. It has been shown that coupling a mechanical oscillator to three optical modes spaced by its mechanical resonance frequency allows overcoming this limitation [84]. Pumping the central resonance, the motional sidebands at $\pm \Omega_{\mathrm{m}}$ are resonant with the adjacent optical modes, enhancing the transduction of motion. Thus, $P_{S Q L}$ may be lower by a factor $\times \kappa^{2} /\left(4 \Omega_{\mathrm{m}}^{2}\right)$, which can be considerable. Similarly, if two optical modes spaced by exactly the mechanical resonance frequency are both parametrically coupled to a mechanical mode, the radiation pressure dynamical backaction can be significantly enhanced [48]. When pumping the higher or lower optical resonance, the weight of the Stokes and anti-Stokes sidebands are strongly asymmetric, similar to the resolved sideband case of a single resonance. However, the pump laser is now, in contrast to the resolved sideband case, resonantly coupled to one of the optical resonances and thus the dynamical backaction is enhanced by a factor $\sim 2 \Omega_{\mathrm{m}}^{2} / \kappa^{2}$. This may allow a drastic reduction of the power levels needed for ground-state cooling.

Here, the feasibility of dual optical mode, or three-mode interactions - two optical modes coupled to one mechanical mode - using toroid microresonators is shown. Thereby, the fact that clock- and counterclockwise propagating modes in 

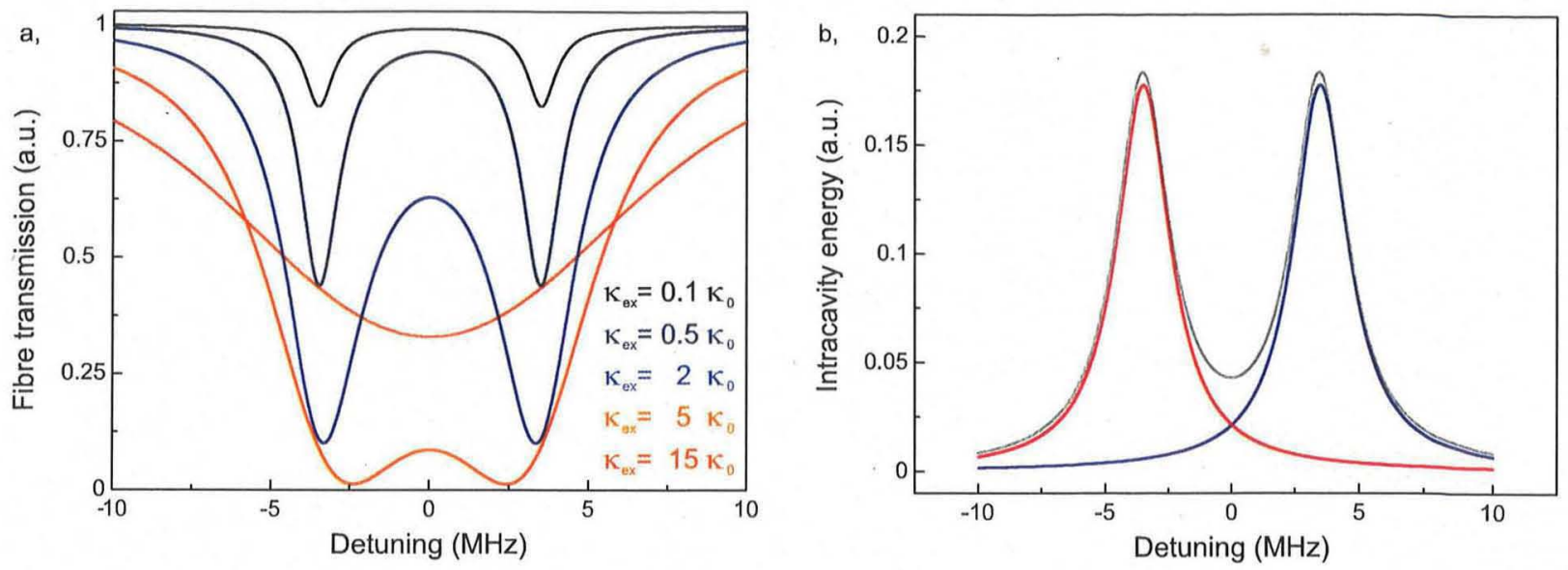

Fig. 10. Mode doublet of a WGM resonator. a, Transmission of a $\kappa_{0} / 2 \pi=1 \mathrm{MHz}$ mode doublet with $\gamma / 2 \pi=7 \mathrm{MHz}$ for various external coupling $\kappa_{\mathrm{ex}}$, as explained in the text. b, Total intracavity energy $\left|\bar{a}_{\mathrm{cw}}\right|^{2}+\left|\bar{a}_{\mathrm{ccw}}\right|^{2}$ (black) as well as the individual contributions of the hybridized lower frequency $\left|\vec{b}_{1}\right|^{2}$ (red) and higher frequency mode $\left|\vec{b}_{2}\right|^{2}$ (blue) for $\kappa / 2 \pi=2.6 \mathrm{MHz}$ and $\gamma / 2 \pi=7 \mathrm{MHz}$.

toroid microresonators, that are intrinsically degenerate, can be coupled via scattering [87-89] and thus form a doublet is the key ingredient. Before looking at the experimental data, we will first have a look at back-scattering induced mode splitting in whispering gallery mode microresonators.

Following a coupled mode approach the mean fields of the coupled clock- and counterclockwise modes $\hat{a}_{\mathrm{cw}}$ and $\hat{a}_{\mathrm{ccw}}$ may be written as (see e.g. Ref. [88] for further details)

$$
\begin{aligned}
& \bar{a}_{\mathrm{cw}}=\sqrt{\kappa_{\mathrm{ex}}} \frac{i \Delta-\kappa / 2}{\Delta^{2}-\kappa^{2} / 4-\gamma^{2} / 4+i \Delta \kappa} \bar{s} \\
& \bar{a}_{\mathrm{ccw}}=-i \frac{\gamma / 2}{i \Delta-\kappa / 2} \bar{a}_{\mathrm{cw}}
\end{aligned}
$$

where we have assumed that only the clockwise mode is pumped via the mean input field $\bar{s}$. The former is, however, also coupled to the counterclockwise mode via the scattering rate $\gamma$. Here, the detuning $\Delta$ is defined with respect to the frequency of the originally degenerate optical modes (for simplicity it is assumed that both modes exhibit the same decay rates $\kappa$ ). Vice versa, only $\bar{a}_{\mathrm{cw}}$ is coupled to the output field $\bar{s}_{\text {out }}$ via $\bar{s}_{\text {out }}=\bar{s}-\sqrt{\kappa_{\mathrm{ex}}} \bar{a}_{\mathrm{cw}}$. The mean output power $\left|\bar{s}_{\text {out }}\right|^{2}$ is shown in Fig. 10a. The population of the mode $\bar{a}_{\mathrm{ccw}}$, in turn, leads to light being partly reflected from the cavity, $\bar{s}_{\mathrm{r}}=-i \kappa_{\mathrm{ex}} \bar{a}_{\mathrm{ccw}}$.

Clearly, the coupling of both modes to each other leads to the formation of a mode doublet. The doublet can be transformed into a pair of symmetric and antisymmetric modes $\hat{b}_{1}=1 / \sqrt{2}\left(\hat{a}_{\mathrm{cw}}+\hat{a}_{\mathrm{ccw}}\right)$ and $\hat{b}_{1}=1 / \sqrt{2}\left(\hat{a}_{\mathrm{cw}}-\hat{a}_{\mathrm{ccw}}\right)$, representing the lower (higher) frequency mode. This is shown in Fig. 10b, along with the total intracavity field energy $\left|\bar{a}_{\mathrm{cw}}\right|^{2}+\left|\bar{a}_{\mathrm{ccw}}\right|^{2}$. Both modes form sine- and cosine-shaped standing wave patterns [71].

Experimentally, coupling an optical mode doublet to a mechanical oscillator, requires some tunability of either the optical splitting frequency or the mechanical oscillator's resonance frequency in order to match both. This is difficult to achieve using systems that incorporate optical and mechanical degrees of freedom within the same element and do not allow tuning optical and mechanical properties independently.

In toroid microresonators, the optical mode splitting is defined by the density of scattering centres within each optical mode's volume [88] which determines the scattering rate $\gamma$. Thus, although the magnitude of typical splitting frequencies can be increased by deliberately implanting defect centers [72], its exact value is random and cannot be tuned in-situ. Thus, the mechanical degree of freedom has to act as the tunable element. Using the near-field approach, this can in fact be readily implemented since the mechanical resonance frequency can be chosen independently. To this end, arrays of nanomechanical oscillators of gradually changing length (and thus resonance frequency) are fabricated. Thus, exactly matching mechanical oscillation frequency and optical mode splitting can be achieved.

Fig. $2 \mathrm{c}$ shows the transmission of a toroid microresonator resonance doublet with a splitting of $\gamma / 2 \pi \sim 7 \mathrm{MHz}$. When tuning the laser to either sideband, the transduction of equivalent cavity frequency noise is expected to be enhanced at frequencies matching the optical splitting frequency $\gamma$. This is shown in Fig. $11 \mathrm{~b}(\gamma / 2 \pi=7 \mathrm{MHz})$. The blue resonance of the optical doublet is pumped with a diode laser (slightly blue-detuned) and the corresponding amplitude noise spectrum is recorded consisting of thermorefractive noise and mechanical noise of both the cavity itself and the nanomechanical oscillator. When the cavity is strongly overcoupled and thus the splitting is removed (cf. Fig. 10a), the thermorefractive noise exhibits the typical shape [29,90,91], as shown in Fig. 11b. When the same measurement is performed in an undercoupled regime, the transduction of thermorefractive frequency noise shows a fast cut-off, due to narrow cavity bandwidth $(\kappa / 2 \pi \sim$ $2 \mathrm{MHz}$ ). However, an apparent peak at $7 \mathrm{MHz}$ is found, which corresponds to the enhancement of frequencies $\sim \gamma / 2 \pi$ due 

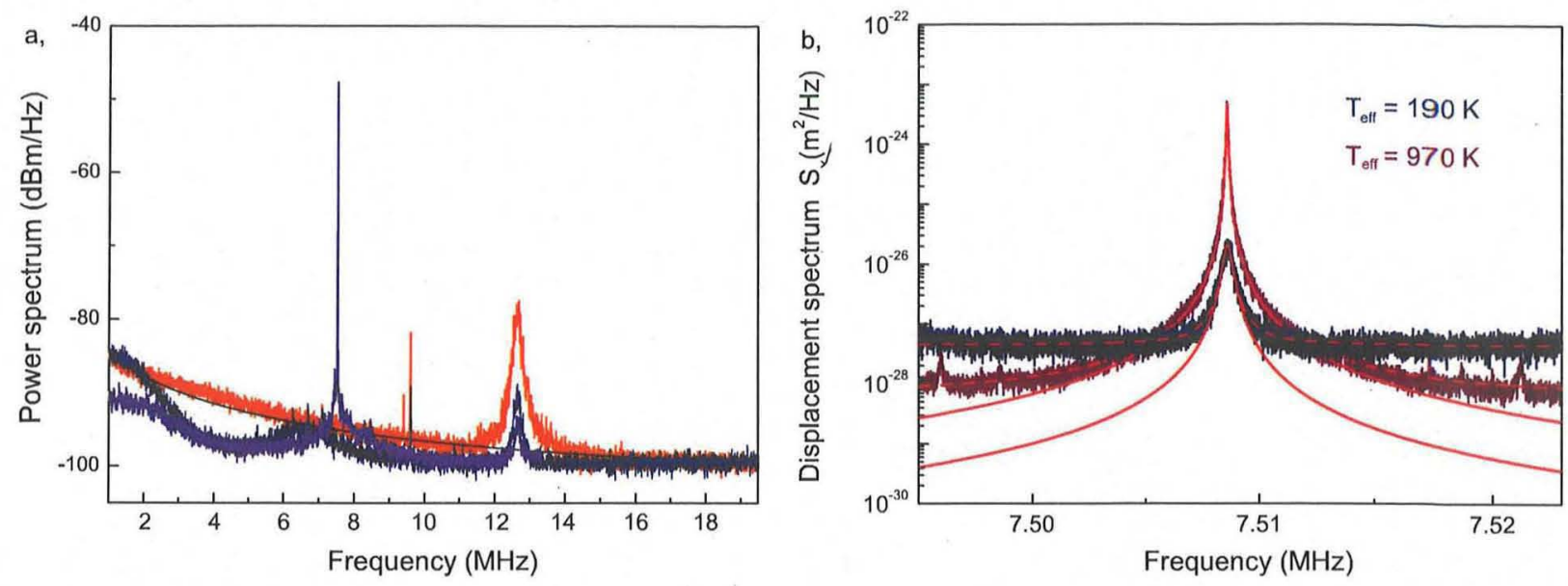

Fig. 11. Three mode interaction. a, Recorded frequency noise spectra. The orange trace is taken in the overcoupled regime ( $g=0$ ). At low frequency, thermorefractive noise dominates, whose magnitude decreases with frequency. The remaining peaks are mechanical modes of the microtoroid. The blue trace is recorded in the undercoupled regime $(g=0)$. The transduction of thermorefractive noise decays faster. It is, however, enhanced for frequencies around $\gamma$. The violet trace is recorded when coupling to a nanomechanical oscillator whose resonance matches the splitting $\gamma$. Since the higher frequency optical mode is used for transduction, the motion of the nanomechanical oscillator is amplified due to dynamical backaction. b, Pumping the red (blue) resonance of an optical doublet (splitting $\gamma / 2 \pi=7 \mathrm{MHz}$ ) leads to dynamical backaction cooling (heating) of the nanomechanical oscillator ( $m_{\text {eff }}=1.6 \times$ $\left.10^{-15} \mathrm{~kg}, \Omega_{\mathrm{m}} / 2 \pi=7.51 \mathrm{MHz}, Q_{\mathrm{m}}=30^{\prime} 000\right)$.

to the presence of the second optical mode. If, in addition, a nanomechanical oscillator $\left(\Omega_{\mathrm{m}} / 2 \pi=7.5 \mathrm{MHz}\right)$ is coupled to the cavity, the degree of coincidence between splitting $\gamma / 2 \pi$ and mechanical frequency $\Omega_{\mathrm{m}} / 2 \pi$ can be directly derived from the recorded RF spectrum. This is shown in Fig. 11b where pumping the blue resonance in addition leads to dynamical backaction, amplifying the nanomechanical oscillator's motion.

The dynamical backaction rate in the case of three-mode coupling can be derived from the photon statistics within the cavity. In Ref. [84], the spectrum of the intracavity photon number fluctuations has been derived for the case of resonantly pumping the red (blue) sideband of a mode doublet. The dynamical backaction rate (for pumping the red and blue sidebands, respectively) can then be calculated

$$
\Gamma_{\mathrm{dba}}^{(2)}= \pm|\bar{a}|^{2} \frac{1}{9+16 \Omega_{\mathrm{m}}^{2} / \kappa^{2}} \frac{64 g_{0}^{2} \Omega_{\mathrm{m}}^{2}}{\kappa^{3}} \stackrel{\Omega_{\mathrm{m}} \gg \kappa}{\approx} \pm 4|\bar{a}|^{2} \frac{g_{0}^{2}}{\kappa}
$$

where the geometric factor describing the overlap between both optical and mechanical modes [48] is neglected for simplicity. If one compares this result to the corresponding limit for a single optical resonance $\left(\Omega_{\mathrm{m}} \gg \kappa\right)$, one arrives at the result that the dynamical backaction rate per intracavity photon is reduced in the two-mode case by a factor $\left(1+16 \Omega_{\mathrm{m}}^{2} / \kappa^{2}\right) /\left(9+16 \Omega_{\mathrm{m}}^{2} / \kappa^{2}\right)$ which approaches unity for $\kappa / \Omega_{\mathrm{m}} \rightarrow 0$. Thus, if the backaction rate is limited by effects due to absorption within the cavity, one does not gain in the regime of two optical modes. One does, however, gain considerably in terms of necessary input power. Since in the two-mode case, the cavity can be pumped resonantly, the dynamical backaction rate can be written as

$$
\Gamma_{\mathrm{dba}}^{(2)} \approx \pm 8 \eta_{\mathrm{c}} \frac{g_{0}^{2}}{\kappa^{2}} \frac{P}{\hbar \omega}
$$

Compared to the single-mode case (cf. Eq. (6)), this corresponds to an enhancement of

$$
\frac{\Gamma_{\mathrm{dba}}^{(2)}}{\Gamma_{\mathrm{dba}}} \sim 2 \Omega_{\mathrm{m}}^{2} / \kappa^{2}
$$

which can be considerable and correspondingly may significantly lower the input power levels necessary for a certain cooling factor. Moreover, the fact that resonant pumping can be used, may facilitate strong cooling from room-temperature since operating in the thermally unstable regime of red detuned locking [80-82] is not necessary, as opposed to the single resonance case. Fig. 11b shows that both cooling and amplification of nanomechanical oscillators is possible using this scheme. This corresponds to the first observation of three mode amplification and cooling of nanomechanical motion. Dynamical backaction rates of order $100 \mathrm{~Hz}$ are achieved. Eq. (26) suggests that considerably stronger backaction rates should in principle be feasible. However, the non-unity overlap of both optical and mechanical modes would have to be taken into account for a quantitative comparison. At least in principle, cooling rates bringing the nanomechanical oscillator close to the quantum ground state even from room temperature are accessible. However, also the limitations owing to the finite mechanical $Q$ factor $\left(Q_{m}>n_{\text {th }} \approx 8 \times 10^{5}\right.$, is required for ground state cooling $\left.[13,14]\right)$ and the thermorefractive noise floor have to be considered in future studies. The latter constraint is a fundamental obstacle, whereas the former may be met by employing mechanical oscillators with larger quality factors $Q_{m}>n_{\text {th }}$ as recently shown [70]. 


\section{Acknowledgements}

T.J.K. acknowledges funding by an ERC Starting Grant (SiMP), a Marie Curie Excellence Grant, the NCCR of Quantum Photonics, DARPA (Orchid programme) and the SNF. The MPQ is gratefully acknowledged for support of this work.

\section{References}

[1] T.J. Kippenberg, K.J. Vahala, Cavity optomechanics: Back-action at the mesoscale, Science 321 (2008) 1172.

[2] F. Marquardt, S.M. Girvin, Optomechanics Physics 2 (2009) 40.

[3] I. Favero, K. Karrai, Optomechanics of deformable optical cavities, Nat. Phot. 3 (2009) 40.

[4] T.J. Kippenberg, et al., Analysis of radiation-pressure induced mechanical oscillation of an optical microcavity, Phys. Rev. Lett. 95 (2005) 033901.

[5] T. Carmon, et al., Temporal behavior of radiation-pressure-induced vibrations of an optical microcavity phonon mode, Phys. Rev. Lett. 94 (2005) 223902.

[6] S. Tallur, et al., Phase noise modeling of opto-mechanical oscillators, in: IEEE Frequency Control Symposium, 2010, pp: 268.

[7] X.L. Feng, et al., A self-sustaining ultrahigh-frequency nanoelectromechanical oscillator, Nat. Nano. 3 (2008) 342.

[8] M. Hossein-Zadeh, et al., Characterization of a radiation-pressure-driven micromechanical oscillator, Phys. Rev. A 74 (2006) 023813.

[9] V.B. Braginskii, A.B. Manukin, M.Y. Tikhonov, Investigation of dissipative ponderomotive effects of electromagnetic radiation, Sov. Phys. JETP 58 (1970) 1550.

[10] O. Arcizet, et al., Radiation-pressure cooling and optomechanical instability of a micromirror, Nature 444 (2006) 71.

[11] S. Gigan, et al., Self-cooling of a micromirror by radiation pressure, Nature 444 (2006) 67.

[12] A. Schliesser, et al., Radiation pressure cooling of a micromechanical oscillator using dynamical backaction, Phys. Rev. Lett. 97 (2006) 243905.

[13] I. Wilson-Rae, et al., Theory of ground state cooling of a mechanical oscillator using dynamical backaction, Phys. Rev. Lett. 99 (2007) 093901.

[14] F. Marquardt, et al., Quantum theory of cavity-assisted sideband cooling of mechanical motion, Phys. Rev. Lett. 99 (2007) 093902.

[15] A. Schliesser, et al., Resolved-sideband cooling of a micromechanical oscillator, Nat. Phys. 4 (2008) 415.

[16] J.D. Thompson, et al., Strong dispersive coupling of a high-finesse cavity to a micromechanical membrane, Nature 452 (2008) 72.

[17] J.D. Teufel, et al., Dynamical backaction of microwave fields on a nanomechanical oscillator, Phys. Rev. Lett. 101 (2008) 197203.

[18] S. Gröblacher, et al., Observation of strong coupling between a micromechanical resonator and an optical cavity field, Nature 460 (2009) 724.

[19] Y.-S. Park, H. Wang, Resolved-sideband and cryogenic cooling of an optomechanical resonator, Nat. Phys. 5 (2009) 489.

[20] A. Schliesser, et al., Resolved-sideband cooling and position measurement of a micromechanical oscillator close to the Heisenberg uncertainty limit, Nat. Phys. 5 (2009) 509.

[21] T. Rocheleau, et al., Preparation and detection of a mechanical resonator near the ground state of motion, Nature 463 (2010) 72.

[22] A.D. O'Connell, et al., Quantum ground state and single-phonon control of a mechanical resonator, Nature 464 (2010) 697.

[23] K. Jensen, K. Kim, A. Zettl, An atomic-resolution nanomechanical mass sensor, Nat. Nano. 3 (2008) 533.

[24] A. Cleland, M. Roukes, A nanometre-scale mechanical electrometer, Nature 392 (1998) 160.

[25] D. Rugar, et al., Single spin detection by magnetic resonance force microscopy, Nature 430 (2004) 329.

[26] A. Abramovici, et al., Improved sensitivity in a gravitational wave interferometer and implications for LIGO, Phys. Lett. A 218 (1996) 157.

[27] Y. Hadjar, et al., High-sensitivity optical measurement of mechanical Brownian motion, Eur. Phys. Lett. 47 (1999) 545.

[28] G. Anetsberger, et al., Near-field cavity optomechanics with nanomechanical oscillators, Nat. Phys. 5 (2009) 909.

[29] G. Anetsberger, et al., Measuring nanomechanical motion with an imprecision below the standard quantum limit, Phys. Rev. A 82 (2010) 061804.

[30] R.G. Knobel, A.N. Cleland, Nanometre-scale displacement sensing using a single electron transistor, Nature 424 (2003) 291.

[31] M.D. LaHaye, et al., Approaching the quantum limit of a nanomechanical resonator, Science 304 (2004) 74.

[32] A. Naik, et al., Cooling a nanomechanical resonator with quantum back-action, Nature 443 (2006) 193.

[33] N.E. Flowers-Jacobs, D.R. Schmidt, K.W. Lehnert, Intrinsic noise properties of atomic point contact displacement detectors, Phys. Rev. Lett. 98 (2007) 096804.

[34] C.A. Regal, J.D. Teufel, K.W. Lehnert, Measuring nanomechanical motion with a microwave cavity interferometer, Nat. Phys. 4 (2008) 555.

[35] S. Etaki, et al., Motion detection of a micromechanical resonator embedded in a d.c. SQUID, Nat. Phys. 4 (2008) 785.

[36] M. Eichenfield, et al., A picogram- and nanometre-scale photonic-crystal optomechanical cavity, Nature 459 (2009) 550.

[37] V.B. Braginsky, S.P. Vyatchanin, On the quantum-non-demolition measurement of the energy of optical quanta, in: Quantum Optics, Experimental Gravity, and Measurement Theory, Plenum Press, 1983.

[38] M.M. Burns, J.-M. Fournier, J.A. Golovchenko, Optical binding, Phys. Rev. Lett. 63 (1989) 1233.

[39] M. Povinelli, et al., High-Q enhancement of attractive and repulsive optical forces between coupled whispering-gallery-mode resonators, Opt. Expr. 13 (2005) 8286.

[40] P.T. Rakich, et al., Trapping, corralling and spectral bonding of optical resonances through optically induced potentials, Nat. Phot. 1 (2007) 658.

[41] M. Eichenfield, et al., Actuation of micro-optomechanical systems via cavity-enhanced optical dipole forces, Nat. Phot. 1 (2007) 416.

[42] A. Ashkin, Optical Trapping and Manipulation of Neutral Particles Using Lasers, World Scientific, Singapore, 2007.

[43] T.W. Hänsch, A.L. Schawlow, Cooling of gases with laser radiation, Opt. Comm. 13 (1975) 68.

[44] D. Van Thourhout, J. Roels, Optomechanical device actuation through the optical gradient force, Nat. Phot. 4 (2010) 211.

[45] J. Teufel, et al., Nanomechanical motion measured with an imprecision below that at the standard quantum limit, Nat. Nano. 4 (2009) 820.

[46] M. Eichenfield, et al., Optomechanical crystals, Nature 462 (2010) 78.

[47] M. Li, et al., Harnessing optical forces in integrated photonic circuits, Nature 456 (2008) 480.

[48] V.B. Braginsky, S.E. Strigin, S.P. Vyatchanin, Parametric oscillatory instability in Fabry-Perot interferometer, Phys. Lett. A 287 (2001) 331.

[49] C.K. Law, Interaction between a moving mirror and radiation pressure: A Hamiltonian formulation, Phys. Rev. A 51 (1995) 2537.

[50] J. Hofer, A. Schliesser, T.J. Kippenberg, Cavity optomechanics with ultrahigh-Q crystalline microresonators, Phys. Rev. A 82 (2010) 031804.

[51] T. Corbitt, et al., An all-optical trap for a gram-scale mirror, Phys. Rev. Lett. 98 (2007) 150802.

[52] S. Gröblacher, et al., Demonstration of an ultracold micro-optomechanical oscillator in a cryogenic cavity, Nat. Phys. 5 (2009) 485.

[53] I. Favero, et al., Optical cooling of a micromirror of wavelength size, Appl. Phys. Lett. 90 (2007) 104101.

[54] G. Anetsberger, et al., Ultralow-dissipation optomechanical resonators on a chip, Nat. Phot. 2 (2008) 627.

[55] Q. Lin, et al., Mechanical oscillation and cooling actuated by the optical gradient force, Phys. Rev. Lett. 103 (2009) 103601.

[56] I. Favero, et al., Fluctuating nanomechanical system in a high finesse optical microcavity, Opt. Expr. 17 (2009) 12813

[57] M. Li, W.H.P. Pernice, H.X. Tang, Reactive cavity optical force on microdisk-coupled nanomechanical beam waveguides, Phys. Rev. Lett. 103 (2009) 223901.

[58] V. Giovannetti, D. Vitali, Phase-noise measurement in a cavity with a movable mirror undergoing quantum Brownian motion, Phys. Rev. A 63 (2001) 023812. 
[59] C.W. Gardiner, M.J. Collett, Input and output in damped quantum systems: Quantum stochastic differential equations and the master equation, Phys. Rev. A 31 (1985) 3761.

[60] A. Dorsel, et al., Optical bistability and mirror confinement induced by radiation pressure, Phys. Rev. Lett. 51 (1983) 1550.

[61] B. Sheard, et al., Observation and characterization of an optical spring, Phys. Rev. A 69 (2004) 051801.

[62] M.L. Gorodetsky, A.E. Fomin, Geometrical theory of whispering-gallery modes, IEEE J. Quant. Electr. 12 (2006) 33.

[63] R. Rivière, et al., Optomechanical sideband cooling of a micromechanical oscillator close to the quantum ground state, arXiv:1011.0290v1 [quant-ph], 2010.

[64] J.G.E. Gardeniers, H.A.C. Tilmans, C.C.G. Visser, LPCVD silicon-rich silicon nitride films for applications in micromechanics, studied with statistical experimental design, J. Vac. Sci. Tech. A 14 (1996) 2879.

[65] S.S. Verbridge, et al., High quality factor resonance at room temperature with nanostrings under high tensile stress, J. Appl. Phys. 99 (2006) 124304.

[66] P.J. French, et al., Optimization of a low-stress silicon nitride process for surface-micromachining applications, Sens. Act. A 58 (1997) 149.

[67] H.J. Lee, et al., Refractive-index dispersion of phosphosilicate glass, thermal oxide, and silicon nitride films on silicon, Appl. Opt. 27 (1988) 4104.

[68] D.P. Poenar, R.F. Wolffenbuttel, Optical properties of thin-film silicon-compatible materials, Appl. Opt. 36 (1997) 5122.

[69] T.J. Kippenberg, S.M. Spillane, K.J. Vahala, Demonstration of ultra-high-Q small mode volume toroid microcavities on a chip, Appl. Phys. Lett. 85 (2004) 6113.

[70] D.J. Wilson, et al., Cavity optomechanics with stoichiometric SiN films, Phys. Rev. Lett. 103 (2009) 207204

[71] A. Mazzei, et al., Controlled coupling of counterpropagating whispering-gallery modes by a single Rayleigh scatterer: A classical problem in a quantum optical light, Phys. Rev. Lett. 99 (2007) 173603.

[72] T.J. Kippenberg, et al., Purcell-factor-enhanced scattering from Si nanocrystals in an optical microcavity, Phys. Rev. Lett. 103 (2009) 027406.

[73] M.L. Gorodetsky, et al., Determination of the vacuum optomechanical coupling rate using frequency noise calibration, Opt. Expr. 18 (2010) 23236.

[74] M. Pinard, Y. Hadjar, A. Heidmann, Effective mass in quantum effects of radiation pressure, Eur. J. Phys. D 7 (1999) 107.

[75] W. Weaver, S.P. Timoshenko, D.H. Young, Vibration Problems Engineering, Wiley and Sons, 1990.

[76] D.R. Southworth, et al., Stress and silicon nitride: A crack in the universal dissipation of glasses, Phys. Rev. Lett. 102 (2009) 225503.

[77] Q.P. Unterreithmeier, T. Faust, J.P. Kotthaus, Damping of nanomechanical resonators, Phys. Rev. Lett. 105 (2010) 027205.

[78] M. Zalalutdinov, et al., Autoparametric optical drive for micromechanical oscillators, Appl. Phys. Lett. 79 (2001) 695.

[79] K.J. Vahala, Back-action limit of linewidth in an optomechanical oscillator, Phys. Rev. A 78 (2008) 023832.

[80] V.B. Braginsky, M.L. Gorodetsky, V.S. Ilchenko, Quality-factor and nonlinear properties of optical whispering-gallery modes, Phys. Rev. A 137 (1989) 393.

[81] V.S. Ilchenko, M.L. Gorodetskii, Thermal nonlinear effects in optical whispering gallery microresonators, Las. Phys. 2 (1992) 1004.

[82] T. Carmon, L. Yang, K.J. Vahala, Dynamical thermal behavior and thermal self-stability of microcavities, Opt. Expr. 12 (2004) 4742.

[83] V.B. Braginsky, S.P. Vyatchanin, Low quantum noise tranquilizer for Fabry-Perot interferometer, Phys. Rev. A 293 (2002) 228.

[84] J.M. Dobrindt, T.J. Kippenberg, Theoretical analysis of mechanical displacement measurement using a multiple cavity mode transducer, Phys. Rev. Lett. 104 (2010) 033901

[85] I.S. Grudinin, et al., Phonon laser action in a tunable two-level system, Phys. Rev. Lett. 104 (2010) 083901.

[86] C. Zhao, et al., Observation of three-mode parametric interactions in long optical cavities, Phys. Rev. A 78 (2008) 023807.

[87] D.S. Weiss, et al., Splitting of high-Q Mie modes induced by light backscattering in silica microspheres, Opt. Lett. 20 (1995) 1835

[88] M.L. Gorodetsky, A.D. Pryamikov, V.S. Ilchenko, Rayleigh scattering in high-Q microspheres, JOSA B 17 (2000) 1051.

[89] T.J. Kippenberg, S.M. Spillane, K.J. Vahala, Modal coupling in traveling-wave resonators, Opt. Lett. 27 (2002) 1669.

[90] M.L. Gorodetsky, I. Grudinin, Fundamental thermal fluctuations in microspheres, JOSA B 21 (2004) 697.

[91] A. Schliesser, et al., High-sensitivity monitoring of micromechanical vibration using optical whispering gallery mode resonators, New J. Phys. 10 (2008) 095015. 